\title{
A hybrid bio-geography based optimization for permutation flow shop scheduling
}

\author{
Minghao Yin $^{1,2}$ and Xiangtao $\mathrm{Li}^{1,2 *}$ \\ ${ }^{1}$ College of Computer Science, Northeast Normal University, Changchun, 130117, P. R. China. \\ ${ }^{2}$ Key Laboratory of Symbolic Computation and Knowledge Engineering of Ministry of Education, Jilin University, 130012, \\ Changchun, P. R. China.
}

Accepted 30 November, 2010

\begin{abstract}
The permutation flow shop problem (PFSSP) is an NP-hard problem of wide engineering and theoretical background. In this paper, a biogeography based optimization (BBO) based on memetic algorithm, named HBBO is proposed for PFSSP. Firstly, to make BBO suitable for PFSSP, a new LRV rule based on random key is introduced to convert the continuous position in BBO to the discrete job permutation. Secondly, the NEH heuristic was combined with the random initialization to initialize the population with certain quality and diversity. Thirdly, a fast local search is used for enhancing the individuals with a certain probability. Fourthly, the pair wise based local search is used to enhance the global optimal solution and help the algorithm to escape from local minimum. Additionally, simulations and comparisons based on PFSSP benchmarks are carried out, showing that our algorithm is both effective and efficient.
\end{abstract}

Key words: Biogeography based optimization, permutation flow shop scheduling, memetic algorithm, local search.

\section{INTRODUCTION}

Scheduling problems play an important role in both manufacturing systems and industrial process for improving the utilization of resources, and therefore it is crucial to develop efficient scheduling technologies (Stadtler, 2005). The permutation flow shop problem (PFSSP), one of the best known production scheduling problems, can be viewed as a simplified version of the flow shop problem, and had been proved to be nondeterministic-polynomial-time (NP)-hard (Garey and Johnson, 1979; Rinnooy, 1976). Due to its significance in both academic and engineering applications, for the permutation flow shop with the criterion of minimizing the makespan or maximum lateness of jobs, different kinds of approaches have been proposed to solve PFSSP and obtained some achievements.

*Corresponding author. E-mail: lixt314@gmail.com. Tel: +860431-84536338. Fax: +86-0431-84536338.
Since the pioneering work of Johnson (Johnson, 1954) on the two machine permutation flow shop problem, many methods have been introduced for solving PFSSP with the objective of minimizing the makespan and minimizing maximum lateness. However, due to unacceptable computation time, exact algorithms such as branch and bound method (Bansal, 1977; Croce et al., 1996; 2002; Ignall and Schrage, 1965) and mixed integer linear programming method (Stafford, 1988) can only solve problems with instances of relatively small size.

Heuristic algorithms were then proposed to solve the large-sized scheduling problems. This kind of algorithms can be broadly classified into three categories: Constructive heuristic algorithms, improvement heuristic algorithms and hybrid heuristic algorithms (Liu and Wang, 2007). The constructive heuristics are mainly simple heuristics that build a feasible scheduling from scratch, as is seen in Palmer (1985), Gupta (1972), Ho and Chang (1991), Campbell et al. (1970), Rajendran (1993), Nawaz et al. (1983), Taillard (1990) and Framinan and 
Leisten (2003). Constructive heuristics usually can obtain a nearly optimal solution in a reasonable computational time, while the solution qualities are not satisfactory.

These heuristics are mainly meta-heuristics that start from previous generated solutions and subsequently approach the optimal solution by improving the solutions with domain dependent knowledge. The meta-heuristics mainly include simulated annealing algorithm (SA) (Ogbu and Smith, 1990; Osman and Potts, 1989), genetic algorithm (GA) (Reeves, 1995; Reeves and Yamada, 1998), artificial immune system algorithm (AIS) (Orhan and Alper, 2004), particle swarm optimization algorithm (PSO) (Tasgetiren et al., 2007), ant colony algorithm (ACO) (Rajendran and Ziegler, 2004; Stützle, 1998), tabu search algorithm (Grabowski and Wodecki, 2004; Nowicki and Smutnicki, 1996; Revees, 1993), iterated local search algorithm(ILS) (Stützle, 1998), estimation of distribution algorithm (EDA) (Bassem and Eddaly, 2009) and biogeography based optimization (Simon, 2008). Improvement heuristics usually can obtain fairly satisfactory solutions, while the solution processes are always time-consuming.

Rather recently, it has become evident that the concentration on a sole meta-heuristic has some limitations. Researchers have found that a skilled combination of two or more meta-heuristic techniques, as called hybrid heuristics, can improve the performance especially when dealing with real-world and large scale problems. A lot of hybrid heuristic based algorithms have been investigated in the past few years. In the paper (Nearchou, 2004), a hybrid SA algorithm was introduced combining the operators of GA with local searches. In the paper (Tseng and Lin, 2009), the genetic algorithm is integrated into a novel local search scheme resulting into two hybrid algorithms: The insertion search and the insertion search with cut-and repair (ISCR).

In the paper (Zhang et al., 2009), two effective heuristics are used during the local search to improve all generated chromosomes in every generation. In Wang and Zhang (2003), Wang Ling used the well-known Nawaz-Enscore-Ham (NEH) combined with GA to generate the initial population, and applied multicrossover operators to enhance the exploring potential. T. Murata proposed a hybrid genetic algorithm with local search (Murata et al., 1996). In the paper (Dipak et al., 2007), a probabilistic hybrid heuristic that combined NEH with SA was proposed for solving PFSSP. In the paper Tasgetiren et al. (2007), applied the PSO algorithm to solve PFSSP by using a small position value rule, and the proposed algorithm, as called PSOVNS, was combined with the variable neighborhood-based local search algorithm. In the paper, Liu and Wang (2007), an efficient particle swarm optimization based mimetic algorithm (MA) for PFSSP to minimize the maximum completion time was proposed. In this algorithm, the PSO-based searching operators and some special local search operators were used to balance the exploration and exploitation abilities. In the paper (Stützle, 1998), an ant-colony based algorithm was proposed to solve PFSSP by combining the fast local search to enhance the solutions. In the paper (Dong and Huang, 2009), a hybrid algorithm that combined FraminanLeisten (FL) heuristic with iterated local search algorithm is proposed.

Regarding minimizing maximum lateness of permutation flow shop scheduling, within our knowledge, only few researchers have used the minimizing maximum lateness as the performance measures of proposed algorithm. In the paper (Tasgetiren et al., 2004), this paper uses the $\mathrm{PSO}_{\mathrm{VNS}}$ to find the optimal solution. This algorithm can find 156 out of 160 upper bounds where 155 of them were improved. In the paper, Zheng and Yamashiro (2010), proposed a new quantum differential evolutionary algorithm. This algorithm based on the basic quantum- inspired evolutionary algorithm (QEA) is encoded and decoded by using the quantum rotating angle and a simple strategy. This algorithm can find 157 out of 160 upper bounds where 156 of them were improved.

Among the existing meta-heuristic algorithms, an evolution technique, biogeography based optimization (Simon, 2008) is a new global optimization algorithm that is simple to be implement and has little or no parameters to be tuned based on biogeography theory, which is the study of the geographical distribution of the biological organisms. The BBO has a way of sharing information which is like other biology based algorithms. One of the remarkable advantages of BBO is that this algorithm can use migration, mutation operators to increase the population diversity. Compared with the 14 benchmarks, $\mathrm{BBO}$ is the fifth faster of the eight algorithm including ACO, BBO, DE, ES, GA, PBIL, PSO, and SGA. Up to now, most published works on BBO mainly focused on solving the complex continuous optimization problem (Ma and Simon, 2010; Ma, 2010; Gong et al., 2010; Bhattacharya and Chattopadhyay, 2010; Bhattacharya and Chattopadhyay, 2010; Roy et al., 2010). Within our knowledge, only few researchers have used the BBO algorithm to solve PFSSP. Therefore, this field of study is still in its early days, a large number of future researches are necessary in order to develop BBO based algorithms for solving PFSSP other than only for those areas the inventors originally focused on.

In this paper, we propose a new hybrid BBO (HBBO) algorithm combing BBO with some local search mechanisms as well as fast local search for solving PFSSP. The crucial idea behind HBBO can be summarized as follows. Firstly, to make HBBO suitable to solve PFSSP, a new LRV rule is proposed based on random key. This rule can help to convert the continuous encoding of BBO to a job permutation. Secondly, The $\mathrm{NEH}$ heuristic was combined with the random initialization 
to initialize the population with certain quality and diversity. Thirdly, multiple different neighborhoods are designed and incorporated as a local search scheme into the searching process to enrich the searching behaviors and to avoid premature convergence. Fast local search is proposed as a component of $\mathrm{BBO}$, for the sake of improving BBO's local search performance. Fourthly, the pair wise based local search is used to enhance the global optimal solution and help the algorithm to escape from local minimum.

\section{PERMUTATION FLOW SHOP SCHEDULING PROBLEM}

The permutation flow shop scheduling problem (PFSSP) in the paper consists of a set of jobs on a set of machines with the objective of minimizing the makespan. In permutation flow shop problem, $\mathrm{n}$ jobs $\mathrm{N}=J_{1}, J_{2}, \ldots, J_{n}$ are to be processed on a series of $m$ machines $\mathrm{M}=M_{1}, M_{2}, \ldots, M_{n}$ sequentially. Each job consists of a set of operations $J_{i}=\left\{O_{i 1}, \ldots, O_{j m}\right\}$. The processing time of job $J_{i}$ on machines $M_{i}$ is denoted by $P_{i, j}(i=1, \ldots, m$, $j=1, \ldots, n)$. Each job can be processed on only one machine at a time and each machine can be processed on only one job at a time. Moreover, the operation cannot be pre-empted. The sequence in which the jobs to be processed are identical for each machine. The objective of the scheduling is to find way to minimize makespan.

The permutation flow shop scheduling problem is often denoted by the symbols $n / m / P / C_{\max }$. A job permutation is denoted by $\pi=\left\{\pi_{1}, \pi_{2}, \ldots, \pi_{n}\right\}$, where $n$ jobs will be sequenced through $\mathrm{m}$ machines. Let $C\left(\pi_{j}, m\right)$ denote the completion time of job $\pi_{j}$ on machine $m$. The completion time of the permutation flow shop scheduling problem according to the processing sequence $\pi=\left\{\pi_{1}, \pi_{2}, \ldots, \pi_{n}\right\}$ is shown as follows:

$C(\pi, 1)=p_{\pi_{1}, 1}$,

$\mathrm{C}\left(\pi_{j}, 1\right)=\mathrm{C}\left(\pi_{j-1}, 1\right)+p_{\pi, 1}, \mathrm{j}=2, \ldots, n$

$\mathrm{C}\left(\pi_{1}, i\right)=\mathrm{C}\left(\pi_{1}, i-1\right)+p_{\pi, i}, i=2, \ldots, m$

$\left.C\left(\pi_{j}, i\right)=\max C\left(\pi_{j-1}, i\right), C\left(\pi_{j}, i-1\right)\right)+p_{\pi, i}, j=2, \ldots n, i=2, \ldots m$

Then makespan can be defined as:

$C_{\max }(\pi)=C\left(\pi_{n}, m\right)$

The goal of the permutation flow shop problem is to find the most suitable arrangement of $\pi{ }^{*}$ :

$C_{\max }\left(\pi^{*}\right) \leq C\left(\pi_{n}, m\right) \forall \pi \in \Pi$.

As for the flow shop scheduling with the due date constraint, let $L\left(\pi_{j}\right)$ be denoted as the lateness of jobs $\pi_{j}$ and be defined as,

$L\left(\pi_{j}\right)=C\left(\pi_{j}, m\right)-d\left(\pi_{j}\right)$.

Maximum lateness $L_{\max }(\pi)$ of a permutation can be defined as

$L_{\max }(\pi)=\max \left(C\left(\pi_{j}, m\right)-d\left(\pi_{j}\right)\right)$,

where $d\left(\pi_{j}\right)$ is the due date of jobs $\pi_{j}$. The optimal solution $\pi *$ should satisfy the following criterion:

$L_{\max }\left(\pi^{*}\right) \leq L_{\max }(\pi) \quad \forall \pi \in \Pi$

and the sum of flow times of all jobs can be describes as

$C_{\text {sum }}\left(\pi^{*}\right)=\sum_{j=1}^{n} C\left(\pi_{j}, m\right)$,

The optimal solution $\pi^{*}$ should satisfy the following criterion:

$C_{\text {sum }}\left(\pi^{*}\right) \leq C_{\text {sum }}(\pi) \forall \pi \in \Pi$

\section{BIOGEOGRAPHY-BASED OPTIMIZATION}

Biogeography based optimization (Simon, 2008) is a new evolution algorithm developed for the global optimization. It is inspired by the immigration and emigration of species between islands in search of more friendly habitats. Each solution is called an "habitat" with an habitat suitability index (HSI) and represented by an n-dimension real vector. An initial individual of the habitat vectors is randomly generated. Those solutions that are good are considered to be habitats with a high HSI. Those that are poor are considered to be habitats with a low HSI. The high HSI tends to share their features with low HSI. Low $\mathrm{HSI}$ solutions accept a lot of new features from high HSI solutions. In BBO, habitat $\mathrm{H}$ is a vector of $\mathrm{N}$ (SIVs) initialized randomly and then follows migration and mutation step to reach the optimal solution. The new candidate habitat is generated from all of the solution in population by using the migration and mutation operators.

In $\mathrm{BBO}$, the migration strategy is similar to the 


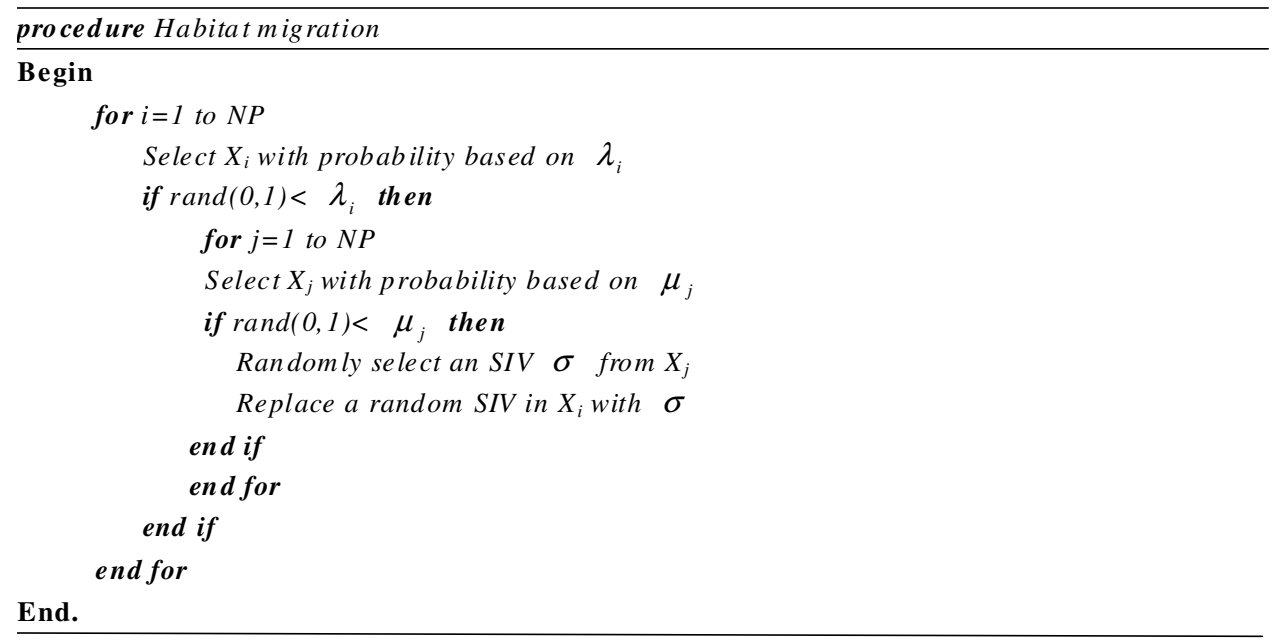

Scheme A. The algorithm of Habitat migration model.

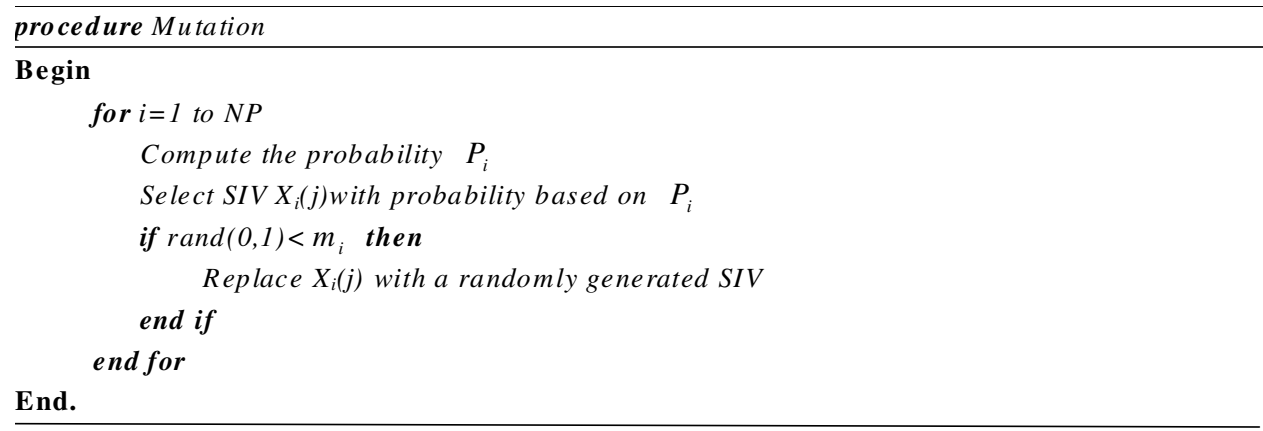

Scheme B. The algorithm of mutation model.

evolutionary strategy in which many parents can contribute to a single offspring. BBO migration is used to change existing solution and modify existing island. Migration is a probabilistic operator that adjusts habitat $X_{i}$. The probability $X_{i}$ modified is proportional to its immigration rate $\lambda_{i}$, and the source of the modified probability from $X_{j}$ is proportional to the emigration rate $\mu_{j}$. Migration can be described as shown in Scheme A.

Mutation is a probabilistic operator that randomly modifies habitat SIVs based on the habitat priori probability of existence. Very high HSI solutions and very low HSI solutions are equally improbable. Medium HSI solutions are relatively probable. The mutation rate $\mathrm{m}$ is expressed as:

$m=m_{\text {max }}\left(\frac{1-P_{s}}{P_{\text {max }}}\right)$

where $m_{\max }$ is a user-defined parameter.
This mutation scheme tends to increase diversity among the population. Mutation can be described as in Scheme $B$. The basic structure of BBO algorithm can be informally described with the algorithm in Scheme C.

\section{BBO for PFSSP}

\section{Solution representation}

In $\mathrm{BBO}$, the standard continuous encoding scheme of BBO can not be used to solve PFSSP directly. In order to apply BBO to PFSSP, one of the key issues is to construct a direct relationship between the job sequences and the vector of individuals in BBO. Qian and wang (2008) proposed a new a largest-order-value (LOV) rule based on random key representation to convert the individual to the job permutation. However, in this paper, we propose a random key based largest-ranked-value (LRV) representation. The LRV rule has the same effect as the LOV rule. Using the LRV rule, we can convert the 


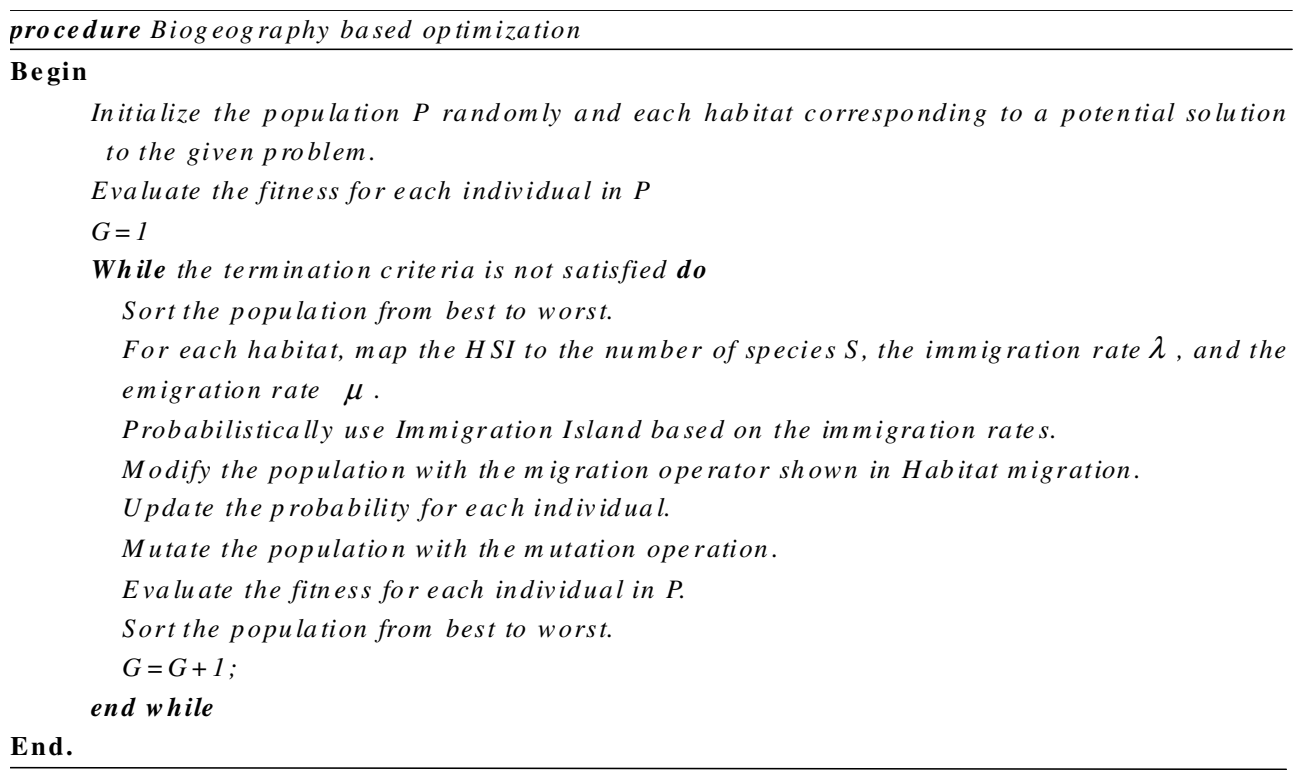

Scheme C. The algorithm of Biogeography based optimization.

Table 1. Solution representation of particle $X_{i}^{t}$.

\begin{tabular}{lcccccc}
\hline Job, dimension & $\mathbf{1}$ & $\mathbf{2}$ & $\mathbf{3}$ & $\mathbf{4}$ & $\mathbf{5}$ & $\mathbf{6}$ \\
\hline Position $x_{i j}^{t}$ & 1.25 & 0.85 & 0.63 & 1.45 & 0.23 & 1.32 \\
Job, $\pi_{i j}^{t}$ & 3 & 4 & 5 & 1 & 6 & 2 \\
\hline
\end{tabular}

continuous real-code vector in BBO to a discrete job permutation. Specifically, in our LRV rule, the largest value of a vector is firstly picked as the first order of a job permutation. After that, the second largest value is picked as the second one. In this way, all the values of the vector will be handled to convert the vector to a job permutation. We use a simple example to illustrate the LRV rule in Table 1. In this example, because the largest value is 1.45 , the dimension $j=4$ is picked and assigned a rank value of one; then the dimension $j=6$ is picked because the second largest value is 1.32 ; in the similar way, the job permutation can be obtained, that is, $\pi=[3,4,5,1,6,2]$. As we can see, such a conversion process is really simple, and it makes BBO applicable for solving PFSSP.

\section{Initial population}

This section will cover the important issues of initialization and follow the subsequent section by the essential concept of implementation. The BBO-based search is applied for exploration. Initial swarm is often generated randomly. In order to enhance the solution, in our paper, we take advantage of the NEH heuristic to produce $10 \%$ vector and the rest of the vectors are initialized with random vector values. Nawaz et al.'s (1983) NEH heuristic is regarded as the best heuristic for the PFSP. The NEH algorithm is based on the idea that the high processing time on all machines should be scheduled as early in the sequence as possible. The NEH heuristic has two phases: (1) The jobs are sorted in non increasing sums of their processing time, and (2) A job sequence is established by evaluating the partial schedules based on the initial order of the first phase. The NEH can be described by the following three steps:

Step 1: Compute the total processing time for each job on $\mathrm{m}$ machine:

$\forall$ job $i, i=1, \cdots, n, P_{i}=\sum_{j=1}^{m} p_{i j}$

Step 2: Sort the jobs in non increasing order of $P_{i}$, then the first two jobs are taken and the two partial possible schedules are evaluated. Choose the better sequence as a current sequence.

Step 3: Take job $i, i=3, \cdots, n$, and find the best schedule by placing it in all possible $i$ positions in the sequence of jobs that are already scheduled. The best sequence would be selected for the next iteration.

The NEH algorithm generated the job permutation, which should be converted to the position values of a certain 


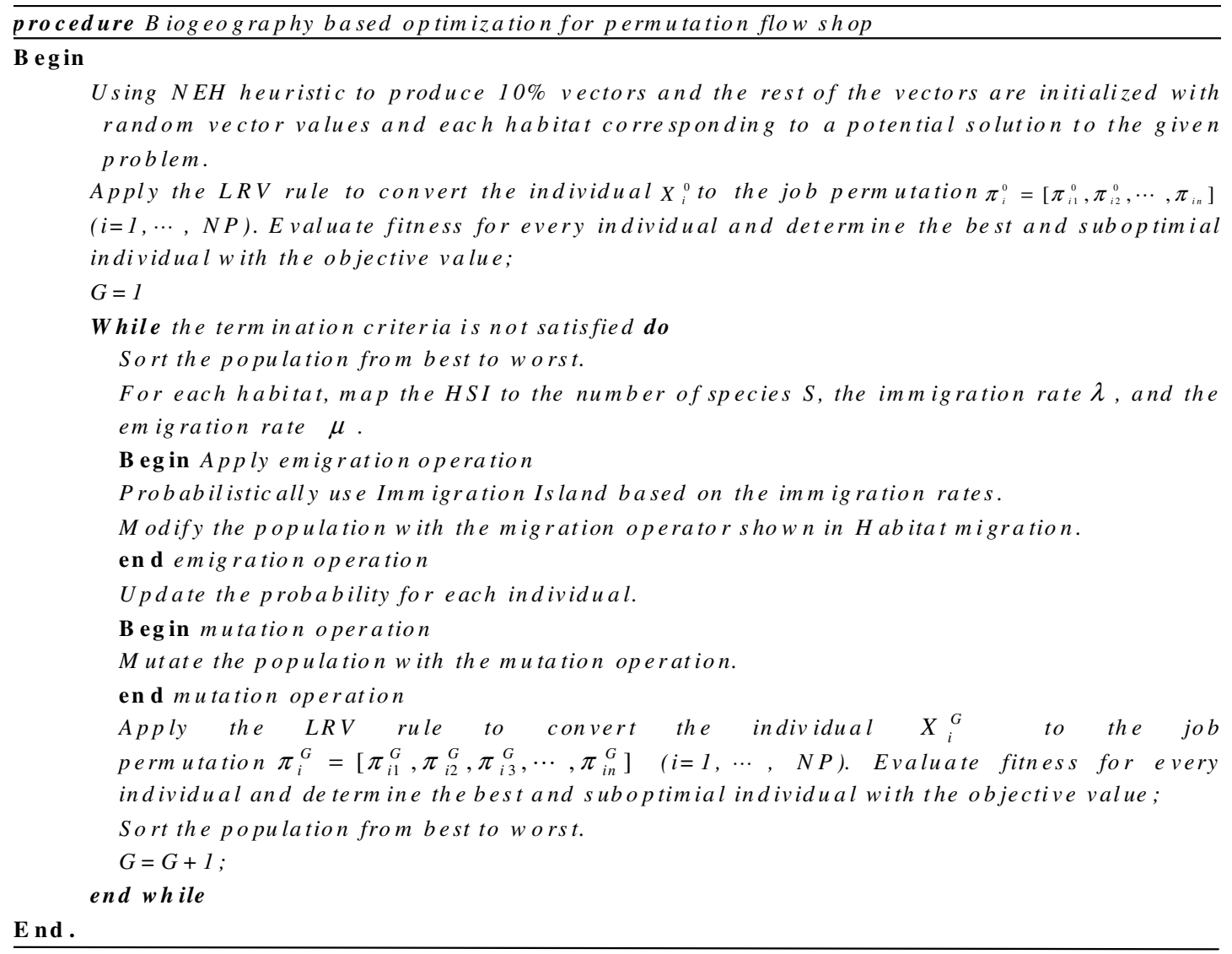

Scheme D. Biogeography based optimization for permutation flow shop scheduling.

individual so that it can adapt to the BBO search. The conversion is implemented using the following equation:

$x_{N E H, i}=x_{\max i}-\frac{\left(x_{\max i}-x_{\min i}\right)}{n} \cdot\left(s q_{N E H, i}-1\right), i=1,2, \cdots, n$

where $x_{N E H, i}$ is the position value in the th dimension of the individual. $s q_{N E H, i}$ is the job index in the ith dimension of the permutation.

The rest of the populations are initialized with random vector values. The initial continuous position values of other vector are first calculated by the following formula:

$x_{i j}^{t}=\left(x_{\max }-x_{\min }\right) * r+x_{\min }$

where $x_{\min }=0, x_{\max }=4.0$ and $r$ is a uniform random number between 0 and 1 .

\section{BBO-BASED SEARCH}

The biogeography based optimization is a population based stochastic optimization algorithm proposed by Simon in 2008 , and the algorithm is similar to the genetic algorithm. The BBO algorithm adopts the real number encoding scheme, migration, mutation based on differential individuals and it has the better ability of overall search ability. Recently, the BBO algorithm was developed to optimize multi-variable and multi-modal continuous functions. Since then, there had been no published work to deal with the permutation flow shop problem by using the biogeography based optimization. However, the BBO algorithm is performed on a continuous space. Therefore, the searching space of the BBO is not the permutation based solution space and we use the LRV rule to convert the continuous individual to the permutation vector. For the initial population, The NEH heuristic combined the random initialization to initialize the population with certain quality and diversity.

The procedure of the biogeography based optimization for PFSSP can be summarized as shown in Scheme d.

\section{Fast local search}

Due to the parallel evolution framework of BBO, local search is easy to be incorporated for exploitation. Here, we present a fast local search which is embedded in BBO for solving PFSSP. The purpose of the local search is to find a better solution from the neighborhood of a solution. In this paper, three neighborhoods, that is, 'Inverse, Insert and Swap' are used to improve the diversity of population and enhance the quality of the solution. The three 

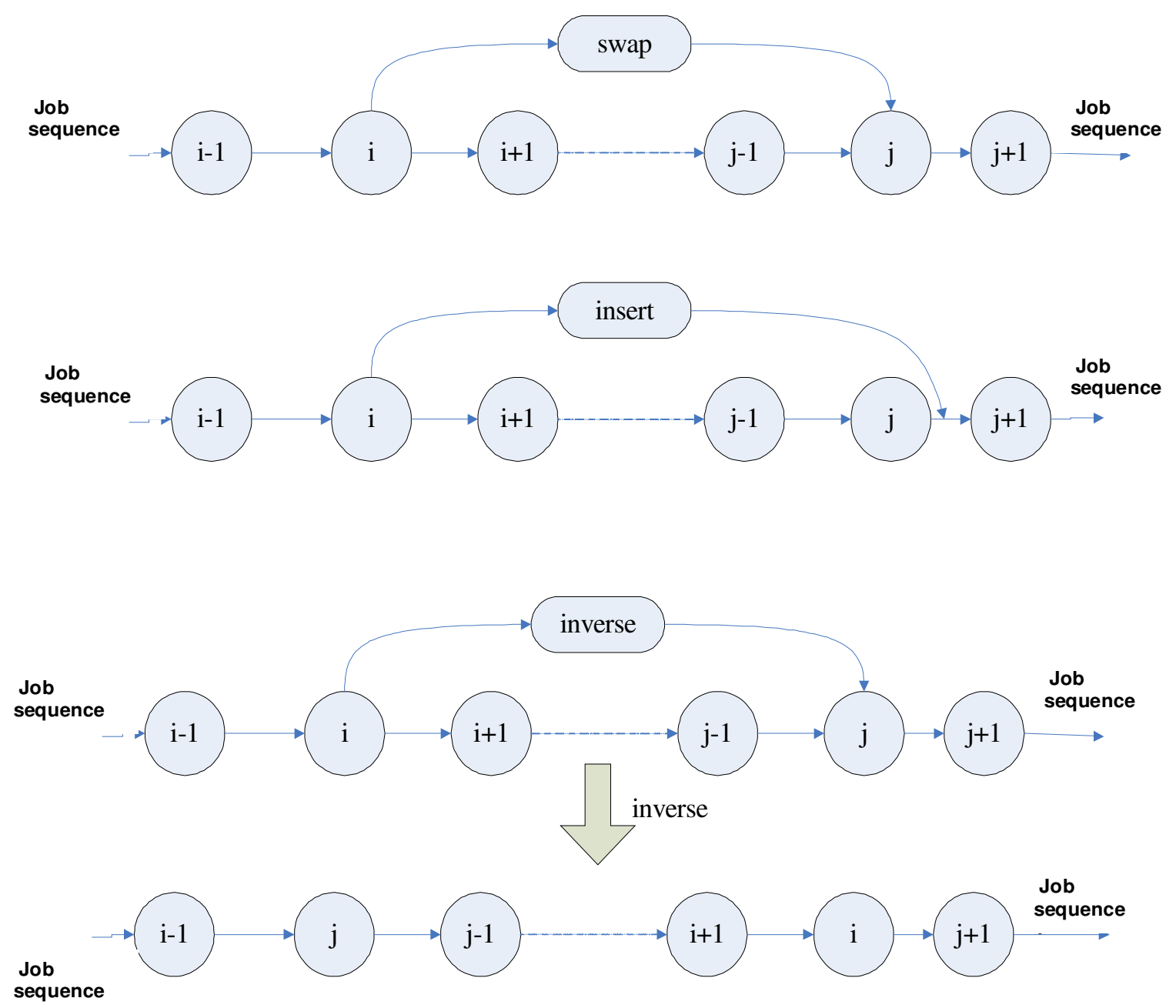

Figure 1. The neighborhood operations for local search.

neighborhood operations are shown in Figure 1. The detail of these neighborhoods is as follows:

Swap: Choose two different positions from a job permutation randomly and swap them. This neighborhood operation is illustrated in Figure 1.

Insert: Choose two different positions from a job permutation randomly and insert the back one before the front. This neighborhood operation is illustrated in Figure 1.

Inverse: Inverse the subsequence between two different random positions of a job permutation. This neighborhood operation is illustrated in Figure 1.

In order to enhance the local search ability and get a better solution, we propose a new fast local search to enhance the makespan of every vector with the certain probability. The algorithm is performed by using the above three operations alternatively to avoid trapping in local optimal points, sometimes.

A new individual enhancement scheme is proposed to combine the insert, swap and inverse operations. This method first selects an operation scheme from the three operations to operate an individual. The selected operation starts from an initial solution and attempts to move from the current solution $x$ to its neighborhood $x^{\prime}$. If the objective fitness of $x^{\prime}$ is smaller than the fitness of the current solution, $x^{\prime}$ is accepted as a new basic solution. After finishing one scheme, the search process keeps generating the individual's neighborhood randomly and the solution is accepted until the stopping criterion is reached (Scheme g).

\section{Remarks}

A partial algorithm about 3 type operations is listed in this algorithm. $p r_{s}$ is the probability of executing swapping operation, $p r_{i}$ is the probability of executing inserting operation, $p r_{i n v}$ is the probability of executing inversing operation.

\section{Pair wise-based local search}

Here, a pair wise-based local search (Liu and Wang, 2007) is employed for the global optimal solution. The crucial idea of pair wise-based local search is to swap the job orders of two adjacent jobs of an individual, and to compare the previous and new 


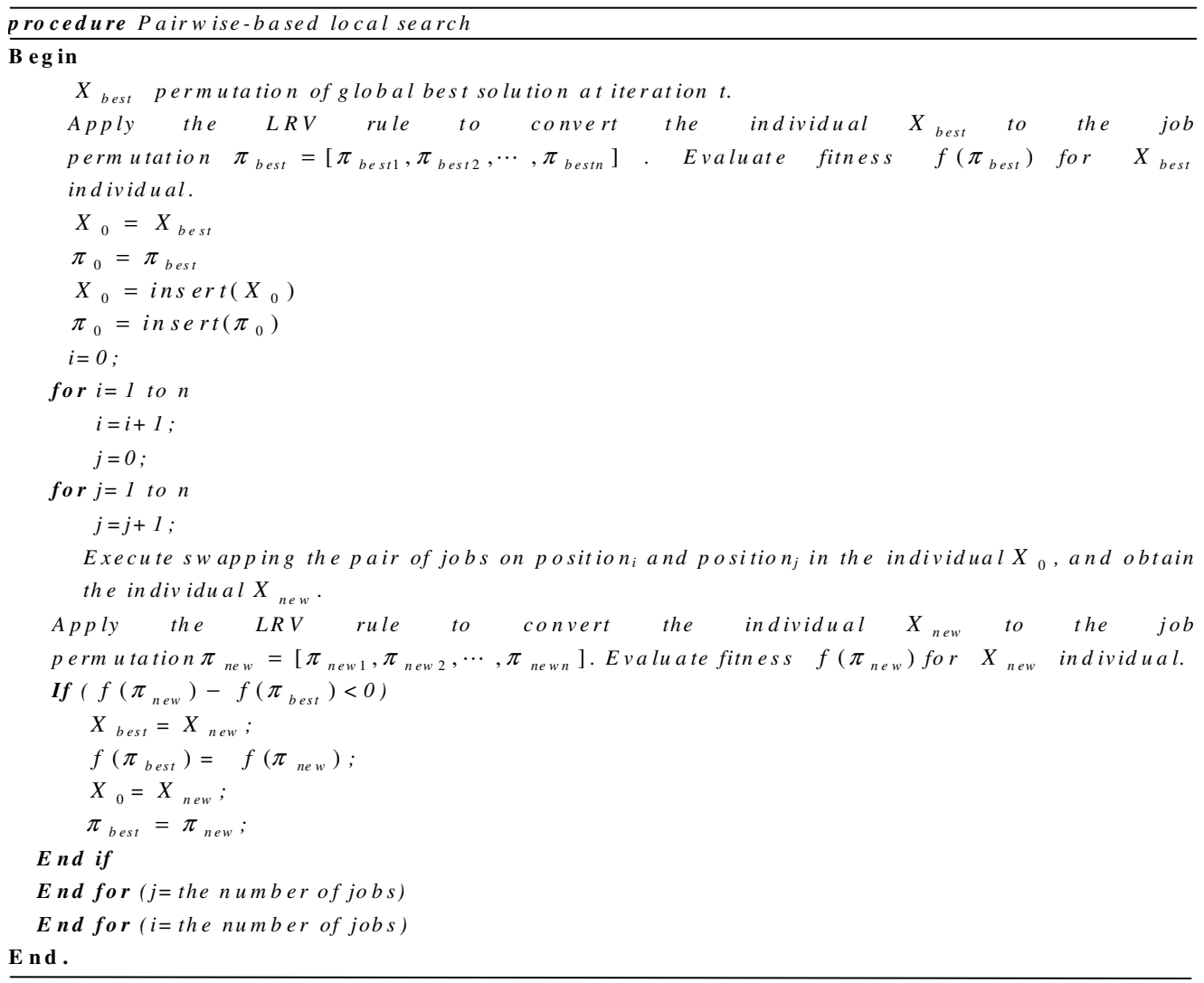

Scheme F. The procedure of the pair wise-based local search algorithm.

Table 2. The execution time for every operation of every job on every machine.

\begin{tabular}{cccccc}
\hline & Job 1 & Job 2 & Job 3 & Job 4 & Job 5 \\
\hline M1 & 3 & 4 & 7 & 8 & 10 \\
M2 & 12 & 15 & 6 & 10 & 8 \\
\hline
\end{tabular}

makespan. If the new makespan is lower than the previous, we will accept the new individual. This method can be also regarded as a local search for BBO to enhance the global optimal solution in every generation. It examines each possible pair wise interchange of the job in the first position. Then other position is given the same operation. Whenever there is an improvement in the objective function, the orders of the jobs are interchanged. Pair wise-based search can be viewed as a detailed neighborhood searching process to enhance the exploitation ability. We will use an example to describe the pair wise-based local search. Table 2 shows the execution time for every operation of every job on every machine. It is assumed that we want to improve the individual $\pi=(5,4,2,1,3)$, whose makespan is 42 and the corresponding Gantt chart is shown in Figure 2. By using the proposed pair wise-based local search, the next individual is $\pi=(4,5,2,1,3)$, whose makespan is 41 and the corresponding Gantt chart is shown in Figure 3. The new individual is lower than the pervious individual. Thus, we will accept the new individual. Table 3 shows the makespans of the example in Table 2.
The procedure of the pair wise-based local search algorithm for PFSSP is as shown in Scheme $\mathrm{f}$.

\section{BBO-based hybrid algorithm}

Here, structure of this hybrid algorithm was discussed. There are mainly four strategies to update the individuals in the proposed algorithm. The LRV rule is used to convert the continuous position in $\mathrm{BBO}$ to the discrete job permutation. The NEH heuristic combined the random initialization to initialize the population with certain quality and diversity. The fast-based local search is used for enhancing the individuals with a certain probability, and therefore has a higher ability to approximate the optimal solution fast. The pair wise based local search is used to enhance the global optimal solution and help the algorithm to escape from local minimum.

Based on the above section, solution representation, initial population, BBO-based local search, fast local search, pair wisebased local search, the procedure of HBBO is proposed as follows:

Step1: Initialize the parameters of BBO algorithm, containing the maximum migration rates $\mathrm{E}$ and $\mathrm{I}$, the maximum rate $m_{\max }$, and the minimal emigration rate $\theta$. Set $\mathrm{G}$ which denotes a generation, $\mathrm{G}=0$. $G_{\max }$ is the evolution generation. NP is the population size. D is the dimension or the number of the jobs. Keep $=2$.

Step 2: Initialize the population, using NEH heuristic to produce $10 \%$ vectors and the rest of the vectors are initialized with random 


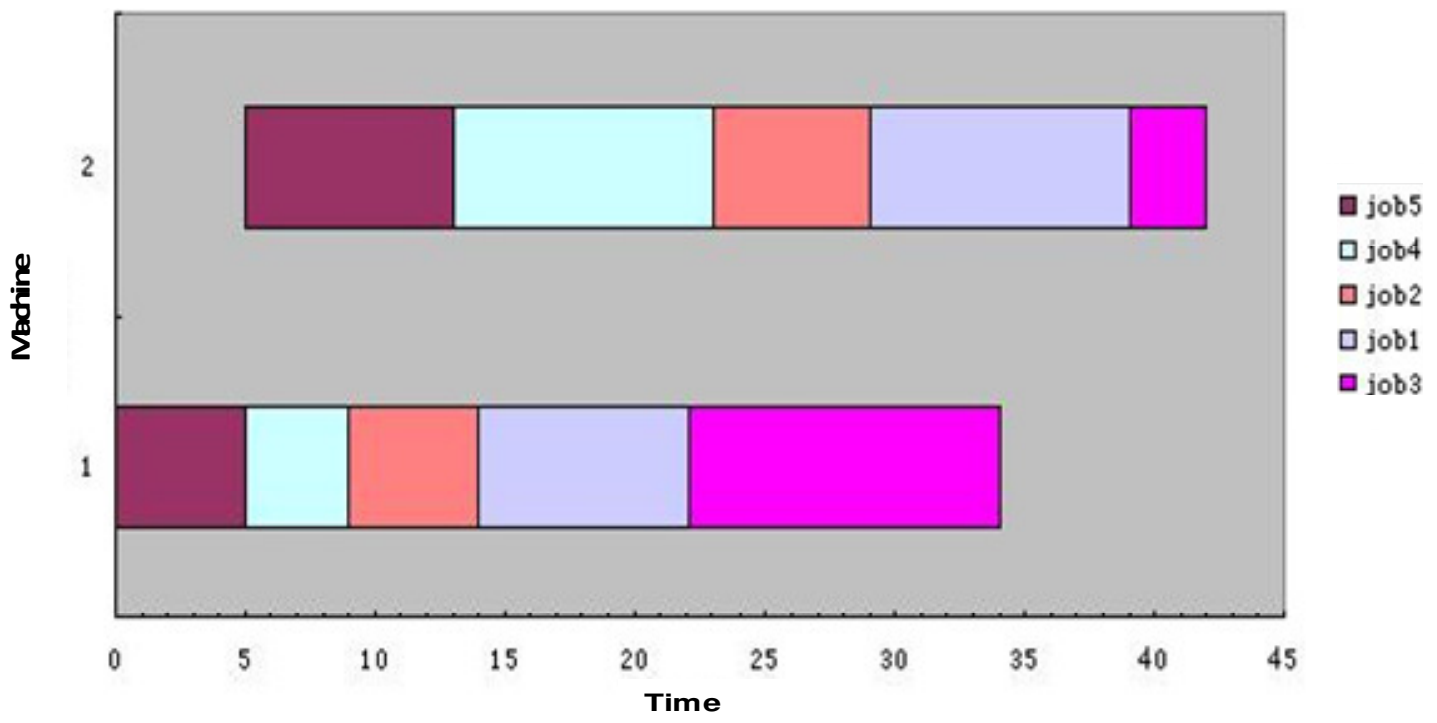

Figure 2. The Gantt chart of the original scheduling.

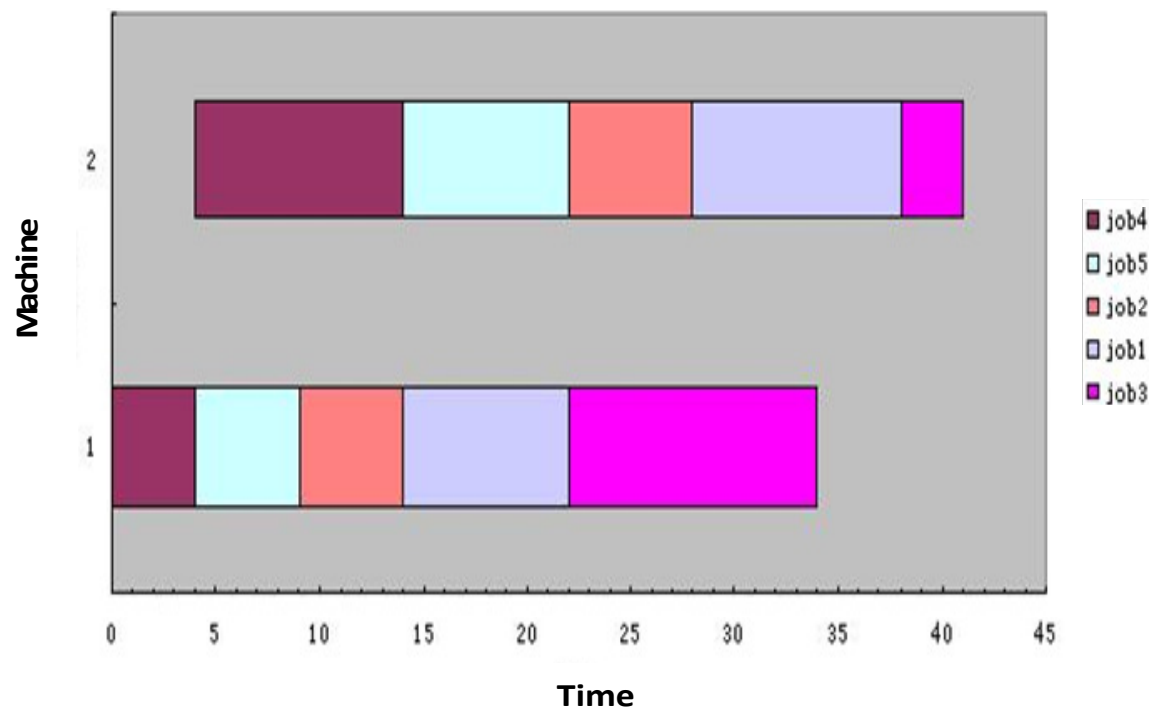

Figure 3. The Gantt chart of the active scheduling.

Table 3. Makespan of the example shown in Table 2.

\begin{tabular}{lccccc}
\hline & Job 1 & Job 2 & Job 3 & Job 4 & Job 5 \\
\cline { 2 - 6 } M1 & 4 & 9 & 14 & 22 & 34 \\
M2 & 14 & 22 & 28 & 38 & 41 \\
\hline
\end{tabular}

vector values and each habitat corresponding to a potential solution to the given problem. Let $x_{\min }=0, x_{\max }=4.0$ Generate $x_{i, j}(0)=x_{\min }+\operatorname{random}(0,1) *\left(x_{\max }-x_{\min }\right)$ $i=1, \cdots, N P, j=1, \cdots, D$.
Step 3: Evaluate the population. Apply the LRV rule to convert the individual $X_{i}^{0}$ to the job permutation $\pi_{i}^{0}=\left[\pi_{i 1}^{0}, \pi_{i 2}^{0}, \pi_{i 3}^{0}, \cdots, \pi_{i n}^{0}\right]_{(i=1, \cdots, N P) \text {. Evaluate fitness for }}$ every individual and sort the fitness from best to worst. Then determine the best and suboptimal individual with the objective value.

Step 4: Perform the evolution.

Step 4.1 (Compute phase): Compute immigration rate $\lambda$ and emigration rate $\mu$ for each species count. $\lambda(i)$ is the immigration rate for habitat $\mathrm{i}, \mu(i)$ is the emigration rate for habitat $\mathrm{i}$.

Step 4.2: (Migration phase): The probability $X_{i}$ modified is 


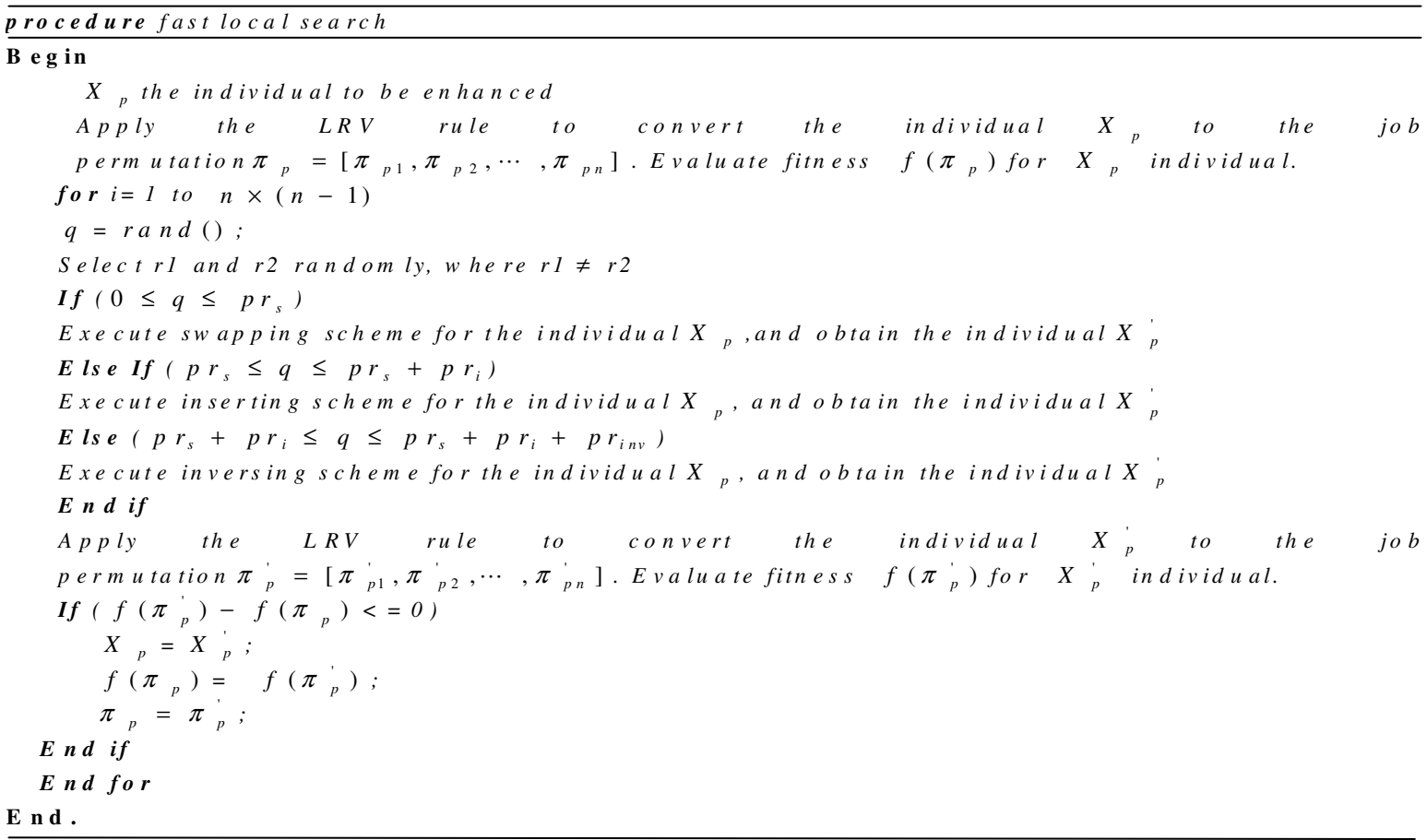

Scheme G. The procedure of the fast local search algorithm.

proportional to its immigration rate $\lambda_{i}$, and the source of the modified probability from $X_{j}$ is proportional to the emigration rate $\mu_{j}$. Step 4.3 (Mutation phase): For each habitat, update the probability of its species count. Then mutate each habitat using the equation $m=m_{\max }\left(\frac{1-P_{s}}{P_{\max }}\right)$ and recompute each habitat's fitness.

Step 4.3 (Compute phase): Make sure that there are no duplicate individuals in the population. Any duplicates that are found are randomly mutated, so there should be a good chance that there are no duplicates in the population.

Step 4.4 (Evaluating phase): Evaluate the population. Apply the LRV rule to convert the individual $X_{i}^{G}$ to the job permutation $\pi_{i}^{G}=\left[\pi_{i 1}^{G}, \pi_{i 2}^{G}, \pi_{i 3}^{G}, \cdots, \pi_{i n}^{G}\right] \quad(\mathrm{i}=1, \cdots, \quad \mathrm{NP})$. Evaluate fitness for every individual and sort the fitness from best to worst. Then determine the best and suboptimal individual with the objective value.

Step 4.5 (Local search phase): Use the fast local search to enhance the best individual, and the pair wise-based local search operations on the suboptimal individual. Through these operations, we save the best one for the next generation.

Step 5 (Stopping criteria): Set $\mathrm{G}=\mathrm{G}+1$. if $\mathrm{G}<G_{\max }$ then go to Step 4 .

\section{NUMERICAL SIMULATION RESULTS AND COMPARISONS}

To test the performance of the proposed HBBO for the permutation flow shop scheduling problem, computational simulations are carried out with some well-studied problems taken from the OR-Library. In this paper, 29 problems from two classes of PFFSP test problems are selected. The first eight problems are instances car1, car2 through to car8 designed by Cariler (1978). The second 21 problems are instances rec01, rec03 through to rec41 designed by Reeves and Yamada (1998). The third 120 instances are from Taillard (1993) and the last problems sets are called DMU problems from Demirkol et al. (1998), containing 160 problems for our experiments. So far, these problems have been widely used as benchmarks to certify the performance of algorithms by many researchers.

The HBBO is coded in MATLAB 7.0, and in our simulation, numerical experiments are performed on a PC with Pentium $3.0 \mathrm{GHz}$ Processor and $1.0 \mathrm{~GB}$ memory. In HBBO, each instance is independently executed 15 times for every algorithm for comparison. The parameters are set as follows: The population size $=30, G_{\max }=300$, mutation probability $\mathrm{P}=0.01$.

\section{Comparisons of BBO, HBBO}

The statistical performances of $\mathrm{BBO}$ and $\mathrm{HBBO}$ are shown in Table 4. In this table, $C^{*}$ is the optimal makespan or lower bound value known so far. BRE 
Table 4. Comparisons of BBO and HBBO.

\begin{tabular}{|c|c|c|c|c|c|c|c|c|c|c|}
\hline \multirow{2}{*}{ Problem } & \multirow{2}{*}{$n / m$} & \multirow[t]{2}{*}{$C^{*}$} & \multicolumn{4}{|c|}{ BBO } & \multicolumn{4}{|c|}{ HBBO } \\
\hline & & & BRE & ARE & WRE & $t_{\text {avg }}$ & BRE & ARE & WRE & $t_{\text {avg }}$ \\
\hline Car1 & $11 / 5$ & 7038 & 0 & 0 & 0 & 0 & 0 & 0 & 0 & 0 \\
\hline Car2 & $13 / 4$ & 7166 & 0 & 0 & 0 & 0.7813 & 0 & 0 & 0 & 0.06 \\
\hline Car3 & $12 / 5$ & 7312 & 0 & 1.0708 & 1.1898 & 0.8125 & 0 & 0 & 0 & 0.26 \\
\hline Car4 & $14 / 4$ & 8003 & 0 & 0 & 0 & 0.0313 & 0 & 0 & 0 & 0 \\
\hline Car5 & $10 / 6$ & 7720 & 0 & 0.9547 & 1.3083 & 0.7656 & 0 & 0 & 0 & 0.03 \\
\hline Car6 & $8 / 9$ & 8505 & 0.7643 & 0.9500 & 2.6220 & 0.5938 & 0 & 0 & 0 & 0.06 \\
\hline Car7 & $7 / 7$ & 6590 & 0 & 0 & 0 & 0 & 0 & 0 & 0 & 0 \\
\hline Car8 & $8 / 8$ & 8366 & 0 & 0 & 0 & 0.1094 & 0 & 0 & 0 & 0.03 \\
\hline Rec01 & $20 / 5$ & 1247 & 0.1604 & 0.8099 & 2.6464 & 1.1563 & 0 & 0.016 & 0.1604 & 11.45 \\
\hline Rec03 & $20 / 5$ & 1109 & 0.6312 & 0.7484 & 1.1722 & 1.1719 & 0 & 0 & 0 & 0.8875 \\
\hline Rec05 & $20 / 5$ & 1242 & 0.2415 & 0.3704 & 0.8857 & 1.1406 & 0.2415 & 0.2415 & 0.2415 & 11.6875 \\
\hline Rec07 & $20 / 10$ & 1566 & 1.1494 & 3.3844 & 3.8314 & 1.2031 & 0 & 0 & 0 & 2.2813 \\
\hline Rec09 & $20 / 10$ & 1537 & 2.2121 & 2.3878 & 2.4073 & 1.1875 & 0 & 0 & 0 & 0.9688 \\
\hline Rec11 & $20 / 10$ & 1431 & 0.2795 & 2.4319 & 3.2145 & 1.2188 & 0 & 0 & 0 & 0.5781 \\
\hline Rec13 & $20 / 15$ & 1930 & 1.2953 & 2.3109 & 2.9534 & 1.2031 & 0 & 0.2383 & 0.9845 & 14.5625 \\
\hline $\operatorname{Rec} 15$ & $20 / 15$ & 1950 & 1.1795 & 1.1846 & 1.2308 & 1.1875 & 0 & 0.1744 & 0.6667 & 14.6563 \\
\hline Rec17 & $20 / 15$ & 1902 & 2.2608 & 3.2282 & 4.5216 & 1.2188 & 0 & 0.2261 & 1.1567 & 14.5313 \\
\hline Rec19 & $30 / 10$ & 2093 & 1.3856 & 2.6899 & 3.7745 & 1.6250 & 0.2867 & 0.4013 & 0.8600 & 32.4219 \\
\hline Rec21 & $30 / 10$ & 2017 & 1.6361 & 1.9683 & 2.8260 & 1.5781 & 0.1487 & 1.2692 & 1.4378 & 32.2344 \\
\hline $\operatorname{Rec} 23$ & $30 / 10$ & 2011 & 2.1382 & 3.1328 & 5.5196 & 1.7031 & 0.3987 & 0.4525 & 0.4973 & 32.2031 \\
\hline $\operatorname{Rec} 25$ & $30 / 15$ & 2513 & 3.2630 & 4.3016 & 5.0537 & 1.6250 & 0 & 0.5412 & 1.1540 & 36.3750 \\
\hline $\operatorname{Rec} 27$ & $30 / 15$ & 2373 & 1.6435 & 2.7729 & 3.5398 & 1.6563 & 0.2107 & 0.4256 & 0.9692 & 36.8281 \\
\hline Rec29 & $30 / 15$ & 2287 & 1.6178 & 3.1351 & 3.9790 & 1.6563 & 0 & 0.7346 & 1.9182 & 36.8750 \\
\hline Rec31 & $50 / 10$ & 3045 & 2.5944 & 3.0049 & 3.3498 & 2.5156 & 0.2627 & 0.4499 & 1.1494 & 108.8438 \\
\hline Rec33 & $50 / 10$ & 3114 & 0.9313 & 0.9891 & 0.9955 & 2.4375 & 0 & 0.4110 & 0.8349 & 108.9063 \\
\hline Rec35 & $50 / 10$ & 3277 & 0.2441 & 0.4333 & 0.7629 & 2.5938 & 0 & 0 & 0 & 3.6719 \\
\hline Rec37 & $75 / 20$ & 4951 & 4.7667 & 5.3282 & 5.9584 & 3.7188 & 1.3533 & 1.7592 & 2.2622 & 368.1250 \\
\hline Rec39 & $75 / 20$ & 5087 & 3.4598 & 4.0849 & 4.4820 & 3.7344 & 0.8649 & 1.2168 & 1.9265 & 365.7188 \\
\hline Rec41 & $75 / 20$ & 4960 & 4.9194 & 5.3750 & 5.7661 & 3.7656 & 1.5121 & 1.9516 & 2.7823 & 370.0625 \\
\hline Average & & & 1.3370 & 1.9672 & 2.5514 & 1.4618 & 0.1820 & 0.3624 & 0.6207 & 55.3210 \\
\hline
\end{tabular}

represents the best relative error to $C^{*}$, ARE denotes the average relative error to $C^{*}$, and WRE represents the worst relative error to $C^{*}$. $t_{\text {avg }}$ Denoted the time to reach the best solution in each run average over $R$ runs in seconds. The performance measures employed in our experiment, BRE, ARE, WRE are defined:

$$
\begin{aligned}
& \text { BRE }=\frac{\text { Heu }_{\text {best }}-\text { Best }_{\text {know }}}{\text { Best }_{\text {know }}} \times 100 \% \\
& \mathrm{ARE}=\sum_{r=1}^{n}\left(\frac{\text { Heu }_{i}-\text { Best }_{\text {know }}}{\text { Best }_{\text {know }}} \times 100\right) \times \frac{1}{n}(\%) \\
& W R E=\frac{\text { Heu }_{\text {worst }_{\text {t }}-\text { Best }_{\text {worst }}}}{\text { Best }_{\text {worst }}} \times 100 \%
\end{aligned}
$$

From Table 4, it can be seen that both HBBOs provide better performance than BBO for almost all benchmarks. The HBBO can provide better solutions than the BBO algorithm. This demonstrates the effectiveness of the fast local search and pairwise-based local search in HBBO. From Table 4, the $t_{\text {avg }}$ of HBBO is more than the BBO. The cause of consuming the time is that the local searches need to consume some time. Additionally, in Table 4, we can also see that, for the problems of the rec01-rec41, the optimal results obtained by the HBBO are closer to $C^{*}$ than BBO. Therefore, in the final HBBO algorithm, these two local searches will be used in the algorithm. From the results, this demonstrates that the global searching ability of HBBO is effective and HBBO is very suitable for PFSSP. To illustrate the experimental results more intuitively, we will set the car06 as an example. 


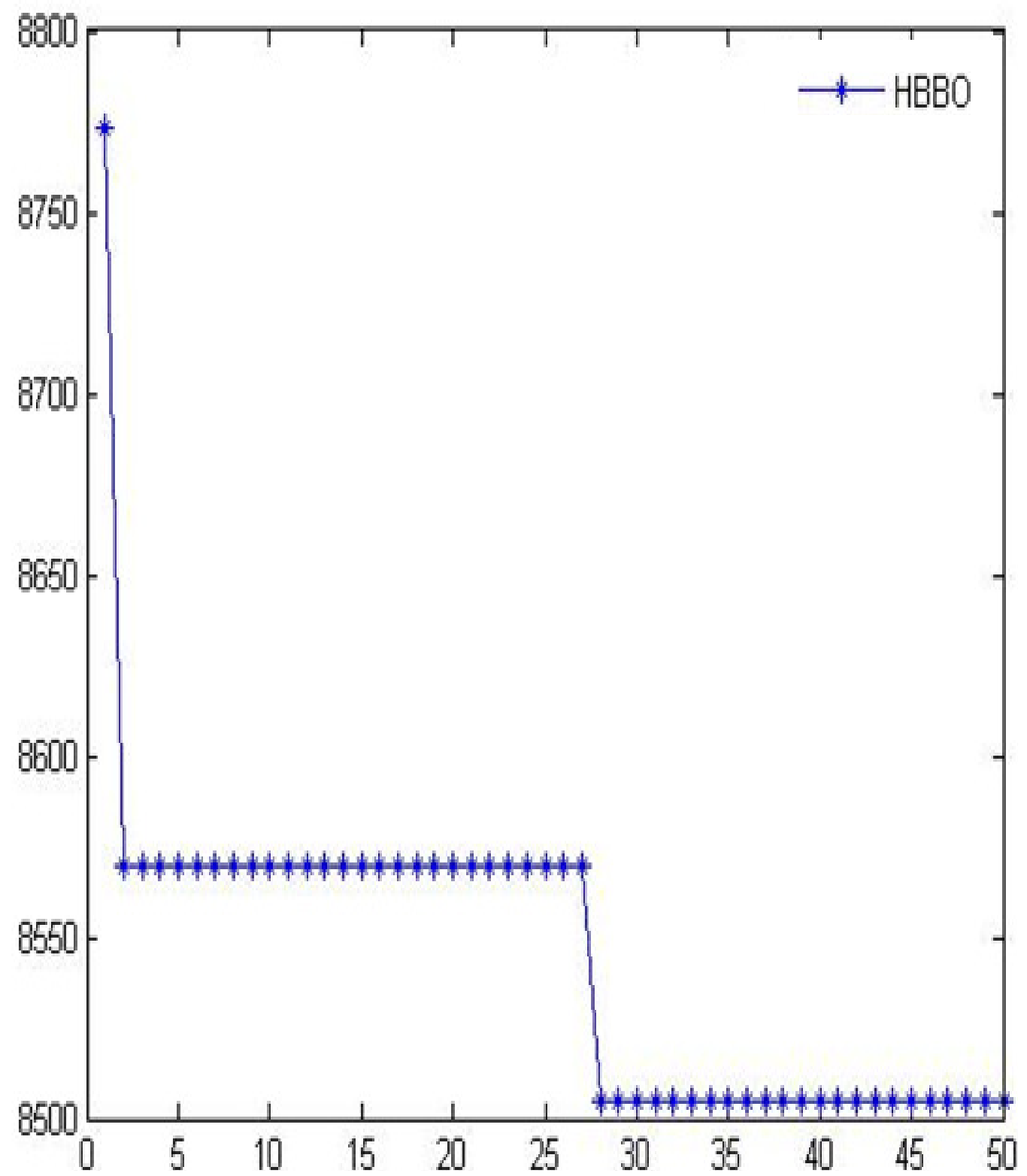

Figure 4. Convergence curves for different algorithms for Car06.

This instance has a lot of local optimal so that it is difficult to find the optimal solution. The convergence rate of HBBO algorithm could be found in Figure 4. It can be seen that at the iteration of $28, \mathrm{HBBO}$ can find the Car's optimal of 8366 .

Comparisons of HBBO, HDE (Qian and wang, 2008) and OSA

In order to show the effectiveness of HBBO, we carry out a simulation to compare our HBBO with another DE based algorithm HDE (Qian and Wang, 2008) and OSA
(Osman and Potts, 1989). HDE applies the parallel evolution mechanism of DE to perform effective exploration (global search), but it also adopts problemdependent local search methodology to adequately perform exploitation (local search). OSA's performance is an efficient algorithm which uses insert to construct the neighborhood. The experimental result is listed in Table 5 . SD denotes the standard deviation of the makespan. From Table 5, it is shown that the BRE, and ARE values obtained by $\mathrm{HBBO}$ are better than those resulting from HDE and OSA for all instances. For the OSA, this algorithm only performs better than the HBBO for instance rec39. For the rest problems, HBBO can provide better 
Table 5. Comparisons of HDE, OSA and HBBO.

\begin{tabular}{|c|c|c|c|c|c|c|c|c|c|}
\hline \multirow{2}{*}{ Problem } & \multicolumn{3}{|c|}{ HDE } & \multicolumn{3}{|c|}{ OSA } & \multicolumn{3}{|c|}{ HBBO } \\
\hline & BRE & ARE & SD & BRE & ARE & SD & BRE & ARE & SD \\
\hline Car1 & 0 & 0 & 0 & 0 & 0 & 0 & 0 & 0 & 0 \\
\hline Car2 & 0 & 0 & 0 & 0 & 0 & 0 & 0 & 0 & 0 \\
\hline Car3 & 0 & 0.536 & 44.352 & 0 & 0.625 & 47.188 & 0 & 0 & 0 \\
\hline Car4 & 0 & 0 & 0 & 0 & 0 & 0 & 0 & 0 & 0 \\
\hline Car5 & 0 & 0.593 & 49.537 & 0 & 0.801 & 50.725 & 0 & 0 & 0 \\
\hline Car6 & 0 & 0.153 & 27.406 & 0 & 2.093 & 274.705 & 0 & 0 & 0 \\
\hline Car7 & 0 & 0.448 & 60.219 & 0 & 1.483 & 114.208 & 0 & 0 & 0 \\
\hline Car8 & 0 & 0 & 0 & 0 & 2.297 & 254.627 & 0 & 0 & 0 \\
\hline Rec01 & 0 & 0.152 & 0.447 & 0.160 & 0.160 & 0 & 0 & 0.016 & 0.6325 \\
\hline $\mathrm{Rec03}$ & 0 & 0.153 & 1.252 & 0 & 0.189 & 1.853 & 0 & 0 & 0 \\
\hline Rec05 & 0.242 & 0.386 & 3.327 & 0.242 & 0.588 & 4.620 & 0.2415 & 0.2415 & 0 \\
\hline Rec07 & 0 & 0.920 & 7.589 & 0 & 0.434 & 11.593 & 0 & 0 & 0 \\
\hline Rec09 & 0 & 0.273 & 11.593 & 0 & 0.690 & 12.385 & 0 & 0 & 0 \\
\hline Rec11 & 0 & 0 & 0 & 0 & 2.215 & 37.600 & 0 & 0 & 0 \\
\hline $\operatorname{Rec} 13$ & 0.259 & 0.705 & 8.566 & 0.311 & 1.793 & 14.691 & 0 & 0.2383 & 5.6411 \\
\hline Rec15 & 0.051 & 0.995 & 14.961 & 0.718 & 1.569 & 16.071 & 0 & 0.1744 & 5.3375 \\
\hline Rec17 & 0.368 & 1.309 & 11.874 & 1.840 & 3.796 & 36.721 & 0 & 0.2261 & 7.0404 \\
\hline Rec19 & 0.287 & 0.908 & 9.104 & 0.287 & 0.803 & 9.484 & 0.2867 & 0.4013 & 5.0596 \\
\hline $\operatorname{Rec} 21$ & 0.198 & 1.284 & 9.171 & 1.438 & 1.477 & 1.687 & 0.1487 & 1.2692 & 8.3293 \\
\hline $\operatorname{Rec} 23$ & 0.497 & 0.696 & 10.625 & 0.497 & 0.845 & 10.822 & 0.3987 & 0.4525 & 0.7379 \\
\hline $\operatorname{Rec} 25$ & 0.676 & 1.429 & 16.489 & 1.194 & 1.938 & 15.063 & 0 & 0.5412 & 8.1131 \\
\hline Rec27 & 0.843 & 1.197 & 4.600 & 0.843 & 1.845 & 21.055 & 0.2107 & 0.4256 & 6.4541 \\
\hline Rec29 & 0.525 & 1.299 & 13.342 & 0.612 & 2.882 & 38.831 & 0 & 0.7346 & 6.6299 \\
\hline $\operatorname{Rec} 31$ & 0.427 & 1.192 & 13.107 & 0.296 & 1.333 & 30.394 & 0.2627 & 0.4499 & 8.0836 \\
\hline Rec33 & 0.353 & 0.787 & 4.743 & 0.128 & 0.732 & 7.315 & 0 & 0.4110 & 12.2638 \\
\hline Rec35 & 0 & 0 & 0 & 0 & 0 & 0 & 0 & 0 & 0 \\
\hline Rec37 & 1.697 & 2.632 & 33.410 & 2.000 & 2.751 & 25.433 & 1.3533 & 1.7592 & 13.6255 \\
\hline Rec39 & 1.278 & 1.543 & 7.836 & 0.767 & 1.240 & 12.306 & 0.8649 & 1.2168 & 18.9529 \\
\hline Rec41 & 1.714 & 2.615 & 36.387 & 1.734 & 2.726 & 39.378 & 1.5121 & 1.9516 & 18.8668 \\
\hline Average & 0.325 & 0.766 & 13.792 & 0.451 & 1.287 & 37.543 & 0.1820 & 0.3624 & 4.3368 \\
\hline
\end{tabular}

solutions. The SD value of the proposed algorithm is also much better than the HDE and OSA for most instances expect Rec01, Rec21, Rec27, Rec33, and Rec 39. It can be concluded that the performance of $\mathrm{HBBO}$ is better than HDE and OSA.

\section{Comparisons of HBBO, PSOMA, PSOVNS}

The performance of HBBO is also compared with other two state-of-art algorithms, that is PSOMA proposed by Bo Liu (Liu and Wang, 2007), and PSOVNS proposed by Tasgetiren (Tasgetiren et al., 2004). We accept the results of those papers and do not ourselves program these algorithms. The experimental result is listed in Table 6. As can be seen in Table 6, the BRE and ARE values obtained by HBBO are much better than PSOMA and PSOVNS. All WRE values obtained by HBBO are better than PSOMA and PSOVNS. It can be concluded that HBBO is more effective than PSOMA and PSOVNS in an acceptable time.

Figure 5 shows the means plot with LSD intervals for the above six algorithms, that is, HDE, OSA, PSOVNS, PSOVNS, BBO and HBBO. Form the results we can see that the HBBO produces statistically better results than all others. Therefore, the HBBO is a robust algorithm for solving PFSSP.

\section{Comparisons of SGA, SGA+NEH, HGA (Wang and Zheng, 2003)}

In order to further show the effectiveness of HBBO, we carry out some comparisons with SGA, HGA and $\mathrm{SGA}+\mathrm{NEH}$ in previous papers. We accept the results of those papers and do not ourselves program these 
Table 6. Comparisons of PSOMA, PSOVNS and HBBO.

\begin{tabular}{|c|c|c|c|c|c|c|c|c|c|}
\hline \multirow{2}{*}{ Problem } & \multicolumn{3}{|c|}{ PSOVNS } & \multicolumn{3}{|c|}{ PSOMA } & \multicolumn{3}{|c|}{ HBBO } \\
\hline & BRE & ARE & WRE & BRE & ARE & WRE & BRE & ARE & WRE \\
\hline Car1 & 0 & 0 & 0 & 0 & 0 & 0 & 0 & 0 & 0 \\
\hline Car2 & 0 & 0 & 0 & 0 & 0 & 0 & 0 & 0 & 0 \\
\hline Car3 & 0 & 0.420 & 1.189 & 0 & 0 & 0 & 0 & 0 & 0 \\
\hline Car4 & 0 & 0 & 0 & 0 & 0 & 0 & 0 & 0 & 0 \\
\hline Car5 & 0 & 0.039 & 0.389 & 0 & 0.018 & 0.375 & 0 & 0 & 0 \\
\hline Car6 & 0 & 0.076 & 0.764 & 0 & 0.114 & 0.764 & 0 & 0 & 0 \\
\hline Car7 & 0 & 0 & 0 & 0 & 0 & 0 & 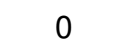 & 0 & 0 \\
\hline Car8 & 0 & 0 & 0 & 0 & 0 & 0 & 0 & 0 & 0 \\
\hline Rec01 & 0.160 & 0.168 & 0.321 & 0 & 0.144 & 0.160 & 0 & 0.016 & 0.1604 \\
\hline $\mathrm{Rec03}$ & 0 & 0.158 & 0.180 & 0 & 0.189 & 0.721 & 0 & 0 & 0 \\
\hline Rec05 & 0.242 & 0.249 & 0.420 & 0.242 & 0.249 & 0.402 & 0.2415 & 0.2415 & 0.2415 \\
\hline $\operatorname{Rec} 07$ & 0.702 & 1.095 & 1.405 & 0 & 0.986 & 1.149 & 0 & 0 & 0 \\
\hline Rec09 & 0 & 0.651 & 1.366 & 0 & 0.621 & 1.691 & 0 & 0 & 0 \\
\hline Rec11 & 0.071 & 1.153 & 2.656 & 0 & 0.129 & 0.978 & 0 & 0 & 0 \\
\hline Rec13 & 1.036 & 1.79 & 2.643 & 0.259 & 0.893 & 1.502 & 0 & 0.2383 & 0.9845 \\
\hline Rec15 & 0.769 & 1.487 & 2.256 & 0.051 & 0.628 & 1.076 & 0 & 0.1744 & 0.6667 \\
\hline Rec17 & 0.999 & 2.453 & 3.365 & 0 & 1.330 & 2.155 & 0 & 0.2261 & 1.1567 \\
\hline Rec19 & 1.529 & 2.099 & 2.532 & 0.43 & 1.313 & 2.102 & 0.2867 & 0.4013 & 0.8600 \\
\hline Rec21 & 1.487 & 1.671 & 2.033 & 1.437 & 1.596 & 1.636 & 0.1487 & 1.2692 & 1.4378 \\
\hline Rec23 & 1.343 & 2.106 & 2.884 & 0.596 & 1.310 & 2.038 & 0.3987 & 0.4525 & 0.4973 \\
\hline Rec25 & 2.388 & 3.166 & 3.780 & 0.835 & 2.085 & 3.233 & 0 & 0.5412 & 1.1540 \\
\hline Rec27 & 1.728 & 2.463 & 3.203 & 1.348 & 1.605 & 2.402 & 0.2107 & 0.4256 & 0.9692 \\
\hline Rec29 & 1.968 & 3.109 & 4.067 & 1.442 & 1.888 & 2.492 & 0 & 0.7346 & 1.9182 \\
\hline Rec31 & 2.594 & 3.232 & 4.237 & 1.510 & 2.254 & 2.692 & 0.2627 & 0.4499 & 1.1494 \\
\hline Rec33 & 0.835 & 1.007 & 1.477 & 0 & 0.645 & 0.834 & 0 & 0.4110 & 0.8349 \\
\hline Rec35 & 0 & 0.038 & 0.092 & 0 & 0 & 0 & 0 & 0 & 0 \\
\hline Rec37 & 4.383 & 4.949 & 5.736 & 2.101 & 3.537 & 4.039 & 1.3533 & 1.7592 & 2.2622 \\
\hline Rec39 & 2.850 & 3.371 & 3.951 & 1.553 & 2.426 & 2.830 & 0.8649 & 1.2168 & 1.9265 \\
\hline Rec41 & 4.173 & 4.867 & 5.585 & 2.641 & 3.684 & 4.052 & 1.5121 & 1.9516 & 2.7823 \\
\hline Average & 1.0089 & 1.4420 & 1.9493 & 0.4981 & 0.9532 & 1.3560 & 0.1820 & 0.3624 & 0.6207 \\
\hline
\end{tabular}

algorithms. SGA is the standard genetic algorithm. HGA is a hybrid genetic algorithm that uses multicrossoveroperators to effect on a subpopulation and uses the SA to enhance it. SGA+NEH is a hybrid genetic algorithm using NEH to improve algorithm performance. The experi-mental results are listed in Table 6 . As can be seen in Table 7, the BRE and ARE values of HBBO are better than those obtained by SGA, SGA+NEH and SGA for all problem expect Rec05. For the HGA, this algorithm only performs better than the HBBO for instance rec05. For the rest problems, HBBO can provide better solutions. Therefore, it can be concluded that HBBO is more effective than these algorithm in an acceptable time.

Figure 6 shows the means plot with LSD intervals for the above five algorithms, that is, SGA, SGA+NEH, HGA, $\mathrm{BBO}$ and $\mathrm{HBBO}$. From the results, we can see that the HBBO produces statistically better results than all others.
Therefore, the HBBO is a robust algorithm for solving PFSSP.

\section{Comparisons of HQEA, QDEA and HBBO}

The performance of HBBO is also compared with other quantum-inspired algorithms. The hybrid quantuminspired evolution algorithm (HQEA) proposed by Wang et al. (2005); the quantum differential evolution algorithm (QDEA) was proposed by Zheng and Yamashiro, (2010). We accept the results of those papers and do not ourselves program these algorithms. The experimental results are listed in Table 8 . From Table 8 , we can find the Car problem, the HQEA, QDEA, HBBO all can find the optimal solution. For the Rec problem, HBBO also can provide better solutions than the other solution 
Table 7. Comparisons of SGA, SGA+NEH, HGA and HBBO.

\begin{tabular}{|c|c|c|c|c|c|c|c|c|}
\hline \multirow{2}{*}{ Problem } & \multicolumn{2}{|c|}{ SGA } & \multicolumn{2}{|c|}{ SGA+NEH } & \multicolumn{2}{|c|}{ HGA } & \multicolumn{2}{|c|}{ HBBO } \\
\hline & BRE & ARE & BRE & ARE & BRE & ARE & BRE & ARE \\
\hline Car1 & 0 & 0.27 & 0 & 0 & 0 & 0 & 0 & 0 \\
\hline Car2 & 0 & 4.07 & 2.93 & 2.93 & 0 & 0 & 0 & 0 \\
\hline Car3 & 1.09 & 2.95 & 0.82 & 1.21 & 0 & 0 & 0 & 0 \\
\hline Car4 & 0 & 2.36 & 0 & 0.07 & 0 & 0 & 0 & 0 \\
\hline Car5 & 0 & 1.46 & 0 & 1.14 & 0 & 0 & 0 & 0 \\
\hline Car6 & 0 & 1.86 & 0 & 2.82 & 0 & 0.04 & 0 & 0 \\
\hline Car7 & 0 & 1.57 & 0 & 1.36 & 0 & 0 & 0 & 0 \\
\hline Car8 & 0 & 2.59 & 0 & 0.03 & 0 & 0 & 0 & 0 \\
\hline Rec01 & 2.81 & 6.96 & 2.25 & 6.13 & 0 & 0.14 & 0 & 0.016 \\
\hline $\operatorname{Rec03}$ & 1.89 & 4.45 & 1.26 & 4.27 & 0 & 0.09 & 0 & 0 \\
\hline Rec05 & 1.93 & 3.82 & 2.33 & 2.90 & 0 & 0.29 & 0.2415 & 0.2415 \\
\hline Rec07 & 1.15 & 5.31 & 3.38 & 5.27 & 0 & 0.69 & 0 & 0 \\
\hline Rec09 & 3.12 & 4.73 & 0.39 & 2.13 & 0 & 0.64 & 0 & 0 \\
\hline Rec11 & 3.91 & 7.39 & 1.19 & 3.66 & 0 & 1.10 & 0 & 0 \\
\hline $\operatorname{Rec} 13$ & 3.68 & 5.97 & 1.92 & 4.41 & 0.36 & 1.68 & 0 & 0.2383 \\
\hline $\operatorname{Rec} 15$ & 2.21 & 4.29 & 2.87 & 4.02 & 0.56 & 1.12 & 0 & 0.1744 \\
\hline Rec17 & 3.15 & 6.08 & 2.16 & 4.02 & 0.95 & 2.32 & 0 & 0.2261 \\
\hline Rec19 & 4.01 & 6.07 & 2.05 & 4.35 & 0.62 & 1.32 & 0.2867 & 0.4013 \\
\hline $\operatorname{Rec} 21$ & 3.42 & 6.07 & 3.52 & 3.58 & 1.44 & 1.57 & 0.1487 & 1.2692 \\
\hline $\operatorname{Rec} 23$ & 3.83 & 7.46 & 3.63 & 5.12 & 0.40 & 0.87 & 0.3987 & 0.4525 \\
\hline Rec25 & 4.42 & 7.20 & 3.14 & 4.89 & 1.27 & 2.54 & 0 & 0.5412 \\
\hline $\operatorname{Rec} 27$ & 4.93 & 6.85 & 3.16 & 5.12 & 1.10 & 1.83 & 0.2107 & 0.4256 \\
\hline $\operatorname{Rec} 29$ & 6.21 & 8.48 & 3.32 & 4.93 & 1.40 & 2.70 & 0 & 0.7346 \\
\hline Rec31 & 6.17 & 8.02 & 5.94 & 6.66 & 0.43 & 1.34 & 0.2627 & 0.4499 \\
\hline Rec33 & 3.08 & 5.12 & 2.70 & 3.38 & 0 & 0.78 & 0 & 0.4110 \\
\hline Rec35 & 1.46 & 3.30 & 1.89 & 2.58 & 0 & 0 & 0 & 0 \\
\hline Rec37 & 7.89 & 10.07 & 7.14 & 7.94 & 3.75 & 4.90 & 1.3533 & 1.7592 \\
\hline Rec39 & 7.32 & 8.51 & 6.25 & 7.09 & 2.20 & 2.79 & 0.8649 & 1.2168 \\
\hline Rec41 & 8.51 & 10.03 & 7.49 & 8.47 & 3.64 & 4.92 & 1.5121 & 1.9516 \\
\hline Average & 2.9721 & 5.2866 & 2.4734 & 3.8097 & 0.6248 & 1.1610 & 0.1820 & 0.3624 \\
\hline
\end{tabular}

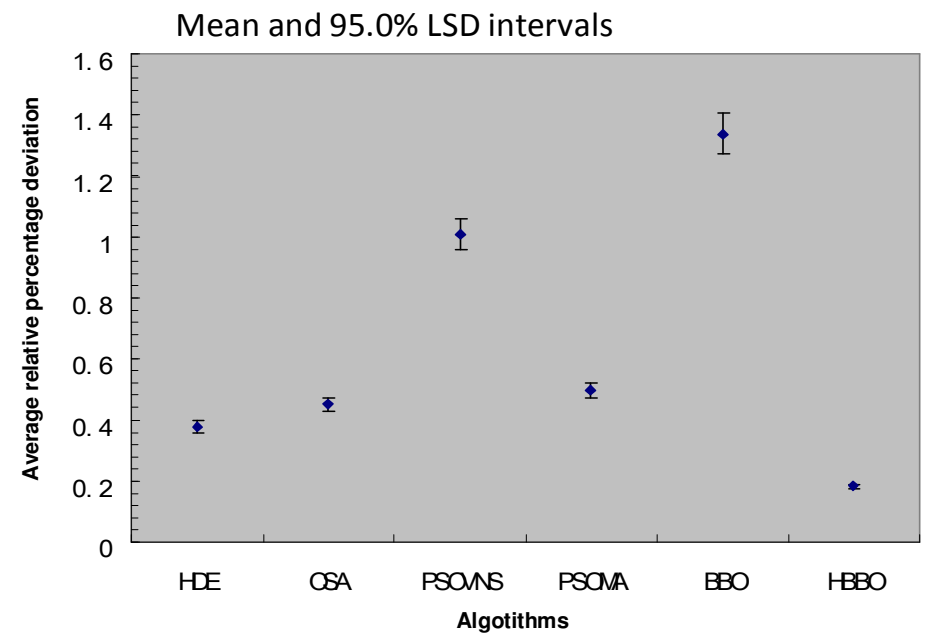

Figure 5. The means plot with LSD intervals for six algorithms (HDE, OSA, PSOVNS, PSOMA, BBO, HBBO). 
Table 8. Comparisons of HQEA, QDEA and HBBO.

\begin{tabular}{lcccccc}
\hline \multirow{2}{*}{ Problem } & \multicolumn{2}{c}{ HQA } & \multicolumn{2}{c}{ QDEA } & \multicolumn{2}{c}{ HBBO } \\
\cline { 2 - 7 } & BRE & ARE & BRE & ARE & BRE & ARE \\
\hline Car1 & 0 & 0 & 0 & 0 & 0 & 0 \\
Car2 & 0 & 0 & 0 & 0 & 0 & 0 \\
Car3 & 0 & 0 & 0 & 0 & 0 & 0 \\
Car4 & 0 & 0 & 0 & 0 & 0 & 0 \\
Car5 & 0 & 0 & 0 & 0 & 0 & 0 \\
Car6 & 0 & 0 & 0 & 0 & 0 & 0 \\
Car7 & 0 & 0 & 0 & 0 & 0 & 0 \\
Car8 & 0 & 0 & 0 & 0 & 0 & 0 \\
Rec1 & 0 & 0.140 & 0 & 0 & 0 & 0.016 \\
Rec03 & 0 & 0.170 & 0 & 0 & 0 & 0 \\
Rec05 & 0.240 & 0.340 & 0.242 & 0.242 & 0.2415 & 0.2415 \\
Rec07 & 0 & 1.020 & 0 & 0 & 0 & 0 \\
Rec09 & 0 & 0.640 & 0 & 0 & 0 & 0 \\
Rec11 & 0 & 0.670 & 0 & 0 & 0 & 0 \\
Rec13 & 0.160 & 1.070 & 0.104 & 0.225 & 0 & 0.2383 \\
Rec15 & 0.050 & 0.970 & 0 & 0.158 & 0 & 0.1744 \\
Rec17 & 0.630 & 1.680 & 0 & 0.126 & 0 & 0.2261 \\
Rec19 & 0.290 & 1.430 & 0.287 & 0.435 & 0.2867 & 0.4013 \\
Rec21 & 1.440 & 1.630 & 0.149 & 1.041 & 0.1487 & 1.2692 \\
Rec23 & 0.500 & 1.200 & 0.348 & 0.597 & 0.3987 & 0.4525 \\
Rec25 & 0.770 & 1.870 & 0.119 & 0.454 & 0 & 0.5412 \\
Rec27 & 0.970 & 1.830 & 0.253 & 0.954 & 0.2107 & 0.4256 \\
Rec29 & 0.350 & 1.970 & 0 & 0.824 & 0 & 0.7346 \\
Rec31 & 1.050 & 2.500 & 0.263 & 0.565 & 0.2627 & 0.4499 \\
Rec33 & 0.830 & 0.910 & 0 & 0.297 & 0 & 0.4110 \\
Rec35 & 0 & 0.150 & 0 & 0 & 0 & 0 \\
Rec37 & 2.520 & 4.330 & 1.717 & 2.771 & 1.3533 & 1.7592 \\
Rec39 & 1.630 & 2.710 & 0.845 & 1.485 & 0.8649 & 1.2168 \\
Rec41 & 3.130 & 4.150 & 1.190 & 1.965 & 1.5121 & 1.9516 \\
Average & 0.502 & 1.082 & 0.1902 & 0.428 & 0.1820 & 0.3624 \\
\hline & & & & & &
\end{tabular}

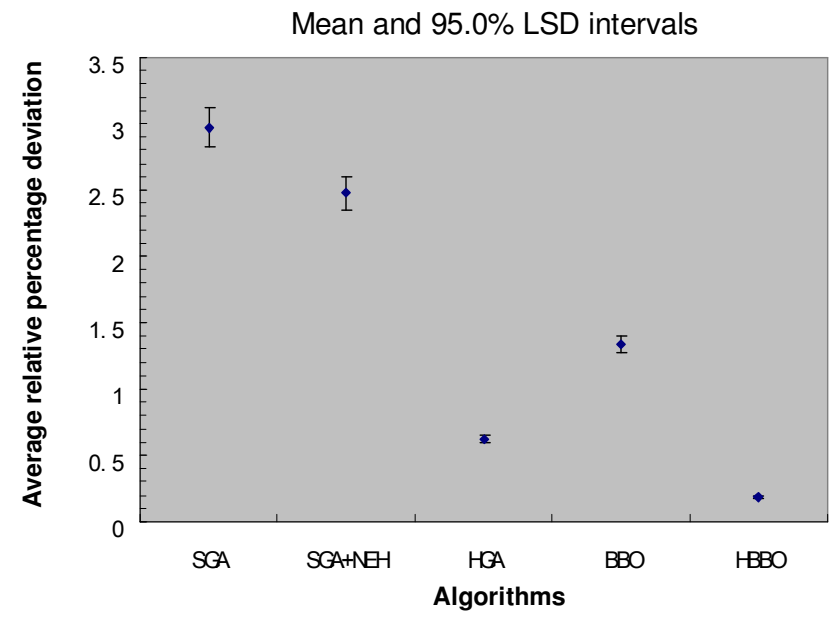

Figure 6. The means plot with LSD intervals for six algorithms (SGA, SGA+NEH, HGA, BBO, HBBO). expect Rec23 and Rec41. For these two problems, our algorithm cannot obtain the better solution than the QDEA. But the ARE of HBBO is better or equal to the QDEA. Therefore, the HBBO algorithm is an effective and robust approach for the PFSP. Figure 7 shows the means plot with LSD intervals for the above algorithms, that is, HQEA, QDEA and HBBO. From the results, we can see that the HBBO produces statistically better results than the other algorithm. Therefore, the HBBO is an effective algorithm for solving PFSSP.

\section{Comparisons on minimizing maximum lateness of PFSP with QDEA}

For the maximum lateness criterion in PFSP, There are two algorithms: PSOVNS (Tasgetiren et al., 2004) and QDEA (Zheng and Yamashiro, 2010). However, the PSOVNS do not report their best solutions in their paper. Therefore, in order to show the effectiveness of HBBO, we carry out a simulation to compare our HBBO algorithms for the minimizing maximum lateness with QDEA. The 160 DMU benchmark problems (Demirkol et al., 1998) (available from http://cobweb.ecn.purdue.edu/ uzsoy2/benchmark/flmax.t $\mathrm{xt})$ are used to demonstrate the algorithm. The experimental solutions are listed in Table 9. We found that PSOVNS can obtain 157 out of 160 upper bounds where 156 of them were improved. However, our HBBO algorithm and QDEA can also obtain 157 out of 160 upper bounds where 156 of them were improved. But our algorithm can find 137 new upper bounds for the DWU problems. For the ARE, the HBBO are all better than the QDEA. The HBBO algorithm provided 137 new upper bounds for future research to provide new algorithms and to compare their results with our solutions.

\section{Effects of the parameter $P$}

Here, we will discuss the effects of the parameter of $P$. The value of $P$ is very important for the BBO algorithm (Simon, 2008). Each individual has an associated probability, which indicates the likelihood that it was expected a priori to exist as a solution to the given problem. If a given problem $S$ has a low probability Ps, then it is surprising that it exists as a solution. It is likely tomutate other solutions. In contrast, a solution with a high probability is likely to mutate to a different solution. Based on it, we also use Car problem and Rec problem as examples to analyze the value of $P$. $P \in\{0.005,0.01,0.02,0.04,0.06,0.08,0.1\}$. The experimental results are listed in Table 10. As can be seen in Table 4, the solution qualities of HBBO vary with $P$, whose value changes from 0.005 to 0.1 .

Figure 8 shows the means plot with LSD intervals for the variant P. From Figure 8, we can clearly see that the 
Mean and $95.0 \%$ LSD intervals

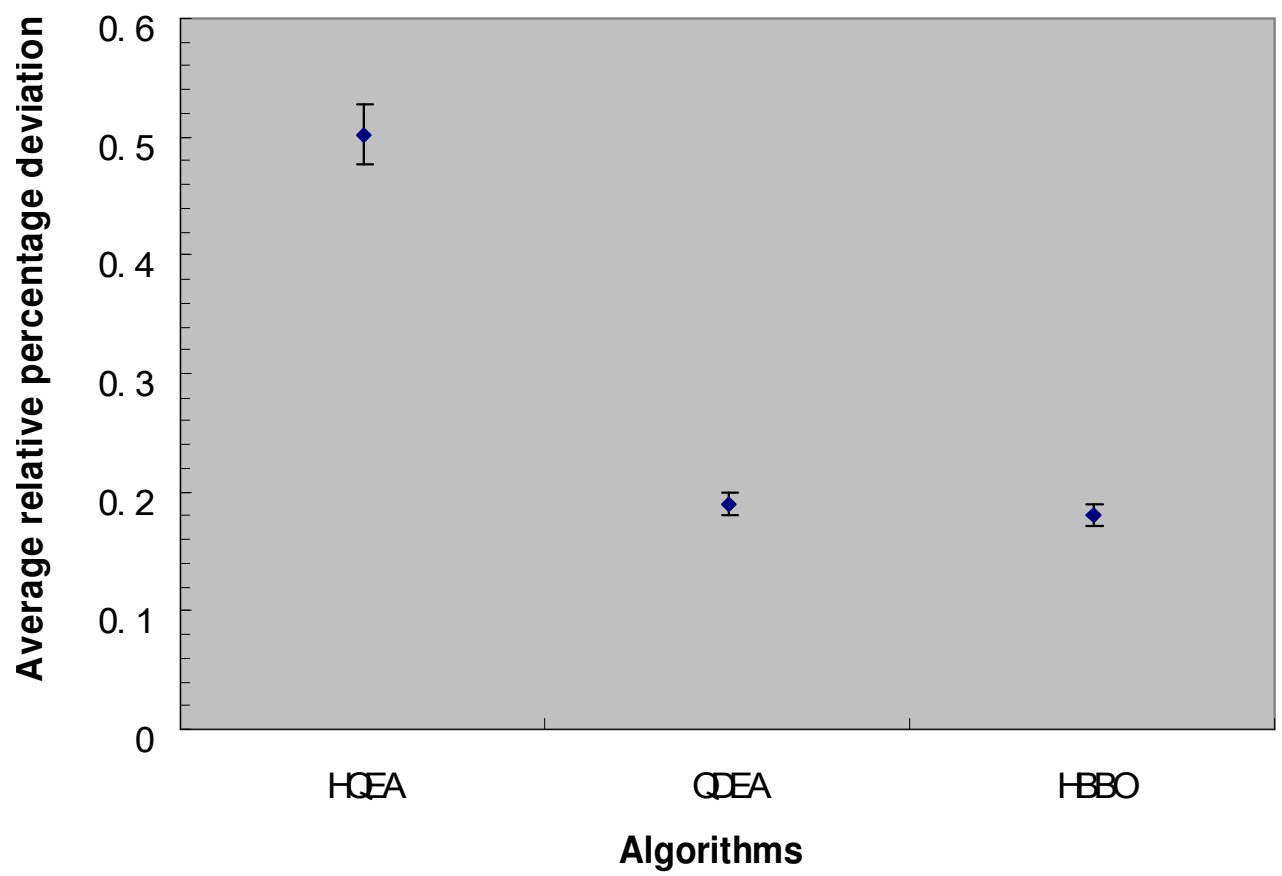

Figure 7. The means plot with LSD intervals for six algorithms.(HQEA, QDEA, HBBO).

Table 9. The maximum lateness obtained by HBBO.

\begin{tabular}{|c|c|c|c|c|c|c|c|c|c|c|c|}
\hline $\mathbf{P}$ & UB & QDEA & HBBO & $\mathbf{P}$ & UB & QDEA & HBBO & $\mathbf{P}$ & UB & QDEA & HBBO \\
\hline \multicolumn{4}{|c|}{20,15} & \multicolumn{4}{|c|}{20,20} & \multicolumn{4}{|c|}{30,15} \\
\hline 1 & 2833 & 2468 & 2431 & 21 & 3437 & 3024 & 2962 & 41 & 2837 & 2291 & 2228 \\
\hline 2 & 2322 & 2087 & 2059 & 22 & 3127 & 2752 & 2748 & 42 & 3088 & 2629 & 2568 \\
\hline 3 & 2370 & 2112 & 2081 & 23 & 2906 & 2745 & 2741 & 43 & 2733 & 2346 & 2270 \\
\hline 4 & 2554 & 2275 & 2255 & 24 & 3197 & 2995 & 2979 & 44 & 3054 & 2689 & 2563 \\
\hline 5 & 2699 & 2330 & 2321 & 25 & 3069 & 2748 & 2715 & 45 & 3074 & 2636 & 2595 \\
\hline 6 & 2239 & 2307 & 2307 & 26 & 2594 & 2579 & 2579 & 46 & 2158 & 2026 & 2007 \\
\hline 7 & 1722 & 1712 & 1712 & 27 & 3388 & 3294 & 3294 & 47 & 1875 & 1748 & 1740 \\
\hline 8 & 2526 & 2508 & 2508 & 28 & 2978 & 2947 & 2947 & 48 & 2637 & 2591 & 2575 \\
\hline 9 & 2165 & 2132 & 2132 & 29 & 2271 & 2210 & 2210 & 49 & 2366 & 2333 & 2288 \\
\hline 10 & 2292 & 2345 & 2340 & 30 & 2836 & 2740 & 2740 & 50 & 2381 & 2368 & 2360 \\
\hline 11 & 3360 & 3042 & 3012 & 31 & 3878 & 3652 & 3631 & 51 & 4465 & 3750 & 3689 \\
\hline 12 & 3651 & 3212 & 3182 & 32 & 3914 & 3564 & 3552 & 52 & 4197 & 3583 & 3501 \\
\hline 13 & 3318 & 2908 & 2890 & 33 & 4076 & 3683 & 3638 & 53 & 3810 & 3236 & 3189 \\
\hline 14 & 3347 & 3092 & 3049 & 34 & 4276 & 3931 & 3901 & 54 & 4472 & 3865 & 3814 \\
\hline 15 & 3251 & 3049 & 3017 & 35 & 3853 & 3580 & 3545 & 55 & 4270 & 3631 & 3562 \\
\hline 16 & 3009 & 2589 & 2563 & 36 & 3231 & 3115 & 3086 & 56 & 3221 & 2792 & 2669 \\
\hline 17 & 2892 & 2627 & 2600 & 37 & 3279 & 3095 & 3053 & 57 & 2983 & 2679 & 2629 \\
\hline 18 & 2462 & 2330 & 2302 & 38 & 3514 & 3364 & 3354 & 58 & 3279 & 2876 & 2815 \\
\hline 19 & 2635 & 2531 & 2518 & 39 & 2998 & 2975 & 2975 & 59 & 3433 & 3053 & 2983 \\
\hline 20 & 2533 & 2457 & 2436 & 40 & 3370 & 3188 & 3188 & 60 & 3252 & 2976 & 2911 \\
\hline APRD & - & -6.953 & -7.6500 & APRD & - & -5.710 & -6.2833 & APRD & - & -11.032 & -12.7970 \\
\hline \multicolumn{4}{|c|}{30,20} & \multicolumn{4}{|c|}{40,15} & \multicolumn{4}{|c|}{40,20} \\
\hline
\end{tabular}


Table 9. Cont'd.

\begin{tabular}{rrrl|rrrr|rrrr}
\hline 61 & 3737 & 3207 & 3091 & 81 & 3530 & 2619 & 2452 & 101 & 4336 & 3495 & 3388 \\
62 & 3592 & 3065 & 3002 & 82 & 3355 & 2662 & 2543 & 102 & 4278 & 3713 & 3564 \\
63 & 4115 & 3496 & 3368 & 83 & 3312 & 2728 & 2588 & 103 & 4216 & 3610 & 3481 \\
64 & 3731 & 3414 & 3333 & 84 & 3060 & 2552 & 2461 & 104 & 4139 & 3682 & 3516 \\
65 & 3254 & 2894 & 2821 & 85 & 3159 & 2691 & 2618 & 105 & 4078 & 3612 & 3474 \\
66 & 3296 & 3191 & 3191 & 86 & 2584 & 2370 & 2337 & 106 & 3379 & 3132 & 3132 \\
67 & 3057 & 2934 & 2934 & 87 & 2343 & 2112 & 2064 & 107 & 3236 & 3212 & 3085 \\
68 & 3158 & 3137 & 3137 & 88 & 2364 & 2364 & 2364 & 108 & 2891 & 2801 & 2779 \\
69 & 3134 & 3166 & 3166 & 89 & 2364 & 2375 & 2375 & 109 & 3627 & 3339 & 3303 \\
70 & 1994 & 1941 & 1893 & 90 & 2503 & 2419 & 2419 & 110 & 2610 & 2505 & 2505 \\
71 & 4472 & 4007 & 3982 & 91 & 5152 & 4426 & 4317 & 111 & 5438 & 4842 & 4678 \\
72 & 4603 & 4199 & 4087 & 92 & 4859 & 3932 & 3850 & 112 & 5640 & 4943 & 4818 \\
73 & 4884 & 4430 & 4332 & 93 & 4969 & 4441 & 4273 & 113 & 5873 & 5066 & 4999 \\
74 & 4628 & 4332 & 4216 & 94 & 4854 & 4123 & 4089 & 114 & 5560 & 4977 & 4885 \\
75 & 4678 & 4117 & 4078 & 95 & 5133 & 4391 & 4297 & 115 & 5536 & 4954 & 4813 \\
76 & 3997 & 3708 & 3657 & 96 & 3596 & 3198 & 3128 & 116 & 4177 & 3643 & 3536 \\
77 & 3721 & 3461 & 3366 & 97 & 3470 & 3245 & 3016 & 117 & 4066 & 3707 & 3608 \\
78 & 3591 & 3370 & 3316 & 98 & 3464 & 3145 & 2970 & 118 & 4590 & 4030 & 3880 \\
79 & 4178 & 3877 & 3843 & 99 & 3479 & 3209 & 3088 & 119 & 3953 & 3757 & 3498 \\
80 & 4111 & 3936 & 3854 & 100 & 3021 & 3021 & 2865 & 120 & 4320 & 3946 & 3795 \\
APRD & - & -7.583 & -9.1465 & APRD & - & -11.640 & -13.8724 & APRD & - & -9.908 & -12.4515 \\
\hline
\end{tabular}

Table 9. Continue.

\begin{tabular}{cccc|cccc}
\hline \multicolumn{4}{c}{$\mathbf{5 0 , 1 5}$} & \multicolumn{4}{|c}{$\mathbf{5 0 , 2 0}$} \\
\hline $\mathrm{P}$ & UB & QDEA & HBBO & P & UB & QDEA & HBBO \\
121 & 4016 & 2976 & 2803 & 41 & 4495 & 3659 & 3524 \\
122 & 3821 & 2935 & 2782 & 42 & 4713 & 3755 & 3614 \\
123 & 3745 & 2774 & 2624 & 43 & 4262 & 3544 & 3475 \\
124 & 3631 & 2774 & 2655 & 44 & 4922 & 3983 & 3834 \\
125 & 3769 & 2942 & 2852 & 45 & 4380 & 3608 & 3435 \\
126 & 2771 & 2602 & 2615 & 46 & 3654 & 3629 & 3536 \\
127 & 2979 & 2972 & 2972 & 47 & 2816 & 2779 & 2734 \\
128 & 3276 & 3064 & 3064 & 48 & 3593 & 3502 & 3487 \\
129 & 2615 & 2606 & 2581 & 49 & 3812 & 3632 & 3611 \\
130 & 3211 & 3190 & 3190 & 50 & 3596 & 3572 & 3506 \\
131 & 5364 & 4554 & 4384 & 51 & 6224 & 5462 & 5349 \\
132 & 5944 & 4765 & 4649 & 52 & 6582 & 5749 & 5591 \\
133 & 5294 & 4540 & 4444 & 53 & 6462 & 5752 & 5593 \\
134 & 5538 & 4659 & 4469 & 54 & 6074 & 5576 & 5368 \\
135 & 5226 & 4516 & 4431 & 55 & 6166 & 5285 & 5179 \\
136 & 3817 & 3433 & 3291 & 56 & 4472 & 4098 & 3941 \\
137 & 3866 & 3473 & 3285 & 57 & 4438 & 4123 & 3986 \\
138 & 3843 & 3483 & 3340 & 58 & 4461 & 4193 & 4002 \\
139 & 4007 & 3233 & 3116 & 59 & 4259 & 3958 & 3839 \\
40 & 3997 & 3806 & 3633 & 60 & 4521 & 4110 & 4009 \\
APRD & - & -13.186 & - & APRD & - & -9.910 & -12.3830 \\
\hline
\end{tabular}


Table 10. The ARE of variant for each algorithm.

\begin{tabular}{|c|c|c|c|c|c|c|c|c|c|c|c|c|c|c|}
\hline \multirow{2}{*}{ Problem } & \multicolumn{2}{|c|}{$P=0.005$} & \multicolumn{2}{|c|}{$P=0.01$} & \multicolumn{2}{|c|}{$P=0.02$} & \multicolumn{2}{|c|}{$P=0.04$} & \multicolumn{2}{|c|}{$P=0.06$} & \multicolumn{2}{|c|}{$P=0.08$} & \multicolumn{2}{|c|}{$P=0.1$} \\
\hline & BRE & ARE & BRE & ARE & BRE & ARE & BRE & ARE & BRE & ARE & BRE & ARE & BRE & ARE \\
\hline Car1 & 0 & 0 & 0 & 0 & 0 & 0 & 0 & 0 & 0 & 0 & 0 & 0 & 0 & 0 \\
\hline Car2 & 0 & 0 & 0 & 0 & 0 & 0 & 0 & 0 & 0 & 0 & 0 & 0 & 0 & 0 \\
\hline Car3 & 0 & 0 & 0 & 0 & 0 & 0 & 0 & 0 & 0 & 0 & 0 & 0 & 0 & 0 \\
\hline Car4 & 0 & 0 & 0 & 0 & 0 & 0 & 0 & 0 & 0 & 0 & 0 & 0 & 0 & 0 \\
\hline Car5 & 0 & 0 & 0 & 0 & 0 & 0 & 0 & 0 & 0 & 0 & 0 & 0 & 0 & 0 \\
\hline Car6 & 0 & 0 & 0 & 0 & 0 & 0 & 0 & 0 & 0 & 0 & 0 & 0 & 0 & 0 \\
\hline Car7 & 0 & 0 & 0 & 0 & 0 & 0 & 0 & 0 & 0 & 0 & 0 & 0 & 0 & 0 \\
\hline Car8 & 0 & 0 & 0 & 0 & 0 & 0 & 0 & 0 & 0 & 0 & 0 & 0 & 0 & 0 \\
\hline Rec01 & 0 & 0.0926 & 0 & 0.016 & 0 & 0.0962 & 0 & 0.0642 & 0 & 0.0642 & 0 & 0.0321 & 0 & 0.0642 \\
\hline $\mathrm{Rec03}$ & 0 & 0 & 0 & 0 & 0 & 0 & 0 & 0 & 0 & 0 & 0 & 0 & 0 & 0 \\
\hline Rec05 & 0.2415 & 0.2415 & 0.2415 & 0.2415 & 0.2415 & 0.2415 & 0.2415 & 0.2415 & 0.2415 & 0.2415 & 0.2415 & 0.2415 & 0.2415 & 0.2415 \\
\hline Rec07 & 0 & 0 & 0 & 0 & 0 & 0 & 0 & 0 & 0 & 0 & 0 & 0 & 0 & 0 \\
\hline Rec09 & 0 & 0 & 0 & 0 & 0 & 0 & 0 & 0 & 0 & 0 & 0 & 0 & 0 & 0 \\
\hline Rec11 & 0 & 0 & 0 & 0 & 0 & 0 & 0 & 0 & 0 & 0 & 0 & 0 & 0 & 0 \\
\hline $\operatorname{Rec} 13$ & 0 & 0.2902 & 0 & 0.2383 & 0.1036 & 0.3005 & 0.1036 & 0.2073 & 0 & 0.1710 & 0 & 0.2850 & 0.1036 & 0.3902 \\
\hline $\operatorname{Rec} 15$ & 0 & 0.4256 & 0 & 0.1744 & 0 & 0.0308 & 0 & 0.0462 & 0 & 0.2154 & 0 & 0.1641 & 0 & 0.1385 \\
\hline $\operatorname{Rec} 17$ & 0 & 0.2366 & 0 & 0.2261 & 0 & 0.2944 & 0 & 0.3891 & 0 & 0.3891 & 0 & 0.2050 & 0 & 0.5100 \\
\hline Rec19 & 0.2867 & 0.4682 & 0.2867 & 0.4013 & 0.2867 & 0.5208 & 0.2867 & 0.5399 & 0.2867 & 0.5495 & 0.2867 & 0.4634 & 0.2867 & 0.6737 \\
\hline Rec21 & 0.1487 & 1.2147 & 0.1487 & 1.2692 & 1.4378 & 1.4576 & 0.5454 & 1.3485 & 0.1487 & 1.1304 & 1.1899 & 1.4328 & 0.8428 & 1.4378 \\
\hline Rec23 & 0.4475 & 0.4923 & 0.3987 & 0.4525 & 0.1492 & 0.4426 & 0.4475 & 0.4873 & 0.4475 & 0.5669 & 0.2486 & 0.4326 & 0.3481 & 0.5619 \\
\hline Rec25 & 0.4437 & 0.9471 & 0 & 0.5412 & 0.3581 & 1.0306 & 0.4775 & 0.8715 & 0.2388 & 0.9272 & 0.2388 & 0.7839 & 0.2786 & 0.9073 \\
\hline Rec27 & 0.6743 & 0.8512 & 0.2107 & 0.4256 & 0.8007 & 0.9987 & 0.2528 & 0.7290 & 0.2528 & 0.9819 & 0.2528 & 0.8133 & 0.2528 & 0.7965 \\
\hline Rec29 & 0.5684 & 0.9969 & 0 & 0.7346 & 0 & 0.7433 & 0.4373 & 0.8920 & 0.3498 & 0.9095 & 0.3061 & 0.7871 & 0 & 0.7346 \\
\hline Rec31 & 0.2956 & 0.4893 & 0.2627 & 0.4499 & 0.3941 & 0.5681 & 0.2956 & 0.4729 & 0.3284 & 0.5583 & 0.2627 & 0.4335 & 0.2627 & 0.4532 \\
\hline Rec33 & 0.1285 & 0.6294 & 0 & 0.4110 & 0 & 0.3597 & 0 & 0.5267 & 0 & 0.5010 & 0 & 0.4624 & 0 & 0.5267 \\
\hline Rec35 & 0 & 0 & 0 & 0 & 0 & 0 & 0 & 0 & 0 & 0 & 0 & 0 & 0 & 0 \\
\hline $\operatorname{Rec} 37$ & 1.9996 & 2.4157 & 1.3533 & 1.7592 & 1.9390 & 2.1733 & 1.9996 & 2.4157 & 2.0400 & 2.3308 & 1.8178 & 2.5207 & 2.0602 & 2.3106 \\
\hline Rec39 & 1.4547 & 1.6906 & 0.8649 & 1.2168 & 1.1205 & 1.4429 & 1.4547 & 1.6906 & 0.9632 & 1.3839 & 1.4940 & 1.7299 & 1.0812 & 1.2227 \\
\hline $\operatorname{Rec} 41$ & 2.0565 & 2.2742 & 1.5121 & 1.9516 & 1.4516 & 2.2661 & 1.4718 & 2.1573 & 2.1976 & 2.3710 & 1.9758 & 2.2621 & 2.2177 & 2.9960 \\
\hline Average & 0.3016 & 0.4743 & 0.1820 & 0.3624 & 0.2856 & 0.4471 & 0.2763 & 0.4510 & 0.2584 & 0.4583 & 0.2867 & 0.4500 & 0.2750 & 0.4816 \\
\hline
\end{tabular}

value of $P$ plays an important role on HBBO, and that when $P$ equals 0.01 , the algorithms can produce statistically better results. Thus, we choose $\mathrm{P}=0.01$ in our algorithm HBBO.
Comparisons of HBBO, BEST (LR), M-MAMAC, PACO and PSO $_{\mathrm{VNS}}$

In order to evaluate the performance of the HBBO algorithm with total flowtime criterion, this algorithm is compared with the performance of these methods proposed by Liu and Reeves,

Rajendran, and Tasgetiren. Liu and Reeves (2001) 


\section{Mean and 95.0\% LSD intervals}

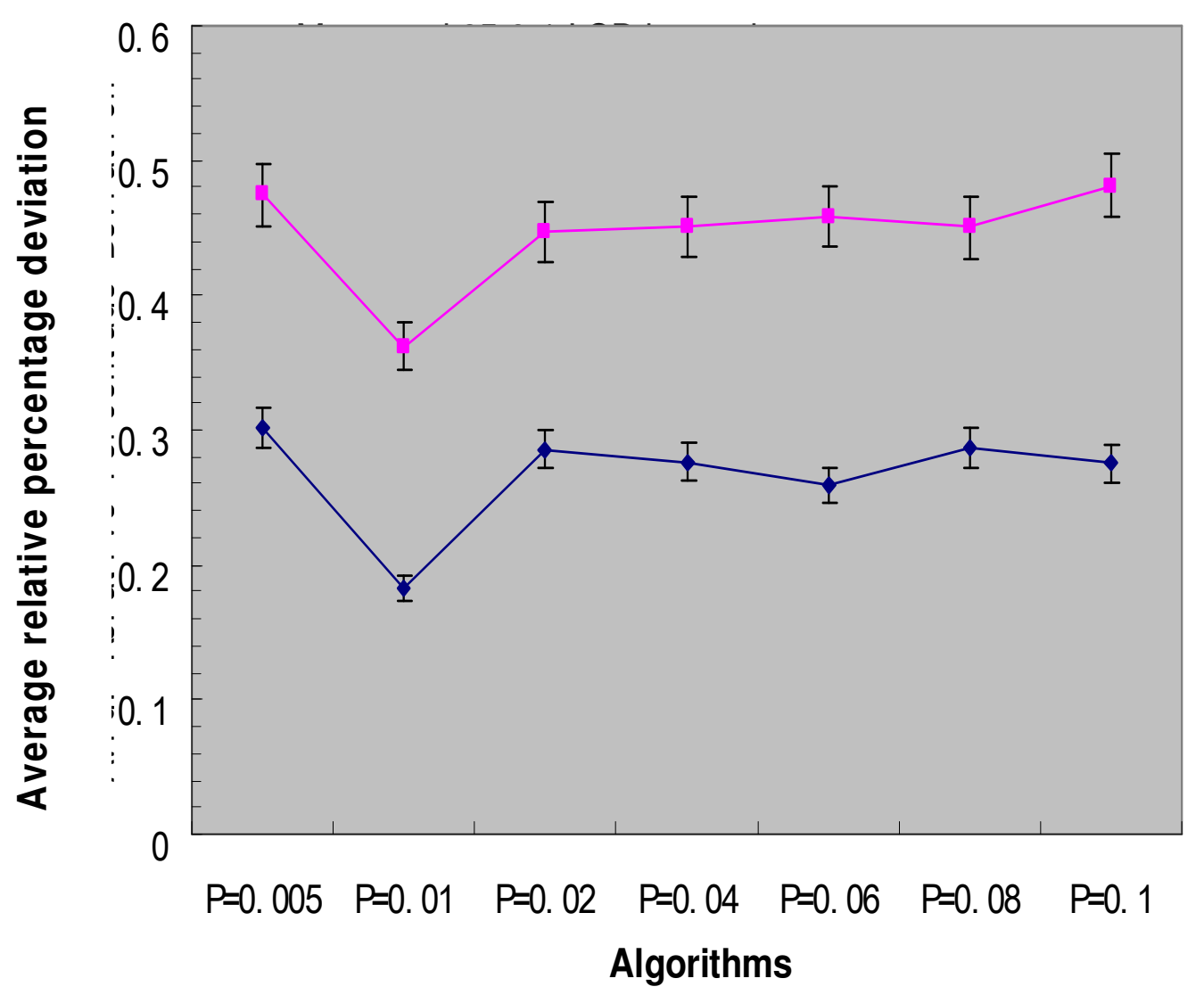

Figure 8. The means plot with LSD intervals for the variant $P$.

developed a couple of new heuristics. This heuristic is one of the best heuristic. Rajendran and Ziegler (2004) developed two new algorithms called M-MMAS and PACO.PSO ${ }_{\text {VNS }}$ (Tasgetiren et al., 2007) presented by the Tasgetiren. The comparative experiments will be carried out on the benchmark problems of Taillard (1993)].

We list the results in Table 11. From Table 11, for the 90 instances considered in this experiment, the HBBO algorithm improves 49 current best solutions. However, the main reason for the success was due to the extensive use of the fast local search and pairwise-based local search in the BBO algorithm where we found that in the first 30 instance, we have 12 instances which have the same solution with other algorithms. For the next 60 instances, $\mathrm{PSO}_{\mathrm{VNS}}$ have 10 instances better than the HBBO algorithm. For the other 50 instances in middle and large scale, the comparison results are all better than the other algorithms which show the global search ability of our method. As the experimental solution shown in Table12, HBBO can provide the better search ability for the large scale problem with the total flow time of jobs.

The experimental results are reported in Table 12.
Further analysis was carried out to see how these algorithms react to the problems. BEST (LR), M-MMAS, $\mathrm{PACO}, \mathrm{PSO}_{\mathrm{VN}}, \mathrm{HBBO}$ are denoted as $\mathrm{P}_{1}, \mathrm{P}_{2}, \mathrm{P}_{3}, \mathrm{P}_{4}$. The APRD is calculated as:

$$
A P R D=\sum_{i=1}^{R}\left(\frac{H_{i}-\min \left(P_{k}, k=1,2,3,4\right) \times 100}{\min \left(P_{k}, k=1,2,3,4\right)}\right) /
$$

The experimental results are listed in Table 12. From the table, it is obvious that the HBBO can provide better solution than the BEST (LR), M-MMAS, PACO, PSOVNS, HBBO, Therefore, the HBBO algorithm provided the new upper bounds which can be used for future research to provide new algorithms; and their results will becompared with our solutions.

\section{Conclusion}

In this paper, a promising hybrid biogeography based optimization is proposed to solve PFSSP. BBO is made 
Table 11. The best solution by HBBO and other four algorithms.

\begin{tabular}{|c|c|c|c|c|c|c|c|c|c|c|c|}
\hline \multirow{2}{*}{$n^{*} m$} & \multicolumn{5}{|c|}{ Total flowtime of jobs } & \multirow{2}{*}{$n^{\star} m$} & \multicolumn{5}{|c|}{ Total flowtime of jobs } \\
\hline & BEST(LR) & M-MMAS & PACO & $\mathrm{PSO}_{\mathrm{VNS}}$ & HBBO & & BEST(LR) & M-MMAS & PACO & PSO $_{\mathrm{VNS}}$ & HBBO \\
\hline \multirow{10}{*}{$20 * 5$} & 14222 & 14056 & 14056 & 14033 & 14033 & \multirow{10}{*}{$50 * 10$} & 88770 & 89599 & 88942 & 88031 & 87654 \\
\hline & 15446 & 15151 & 15214 & 15151 & 15151 & & 85600 & 83612 & 84549 & 83624 & 83555 \\
\hline & 13676 & 13416 & 13403 & 13301 & 13301 & & 82456 & 81655 & 81338 & 80609 & 80322 \\
\hline & 15750 & 15486 & 15505 & 15447 & 15447 & & 89356 & 87924 & 88014 & 87053 & 86921 \\
\hline & 13633 & 13529 & 13529 & 13529 & 13529 & & 88482 & 88826 & 87801 & 87263 & 86984 \\
\hline & 13265 & 13139 & 13123 & 13123 & 13123 & & 89602 & 88394 & 88269 & 87255 & 86816 \\
\hline & 13774 & 13559 & 13674 & 13548 & 13548 & & 91422 & 90686 & 89984 & 89259 & 89517 \\
\hline & 13968 & 13968 & 14042 & 13948 & 13948 & & 89549 & 88595 & 88281 & 87192 & 87607 \\
\hline & 14456 & 14317 & 14383 & 14295 & 14295 & & 88230 & 86975 & 86995 & 86102 & 86015 \\
\hline & 13036 & 12968 & 13021 & 12943 & 12943 & & 90787 & 89470 & 89238 & 88631 & 88783 \\
\hline \multirow{10}{*}{$20 * 10$} & 21207 & 20980 & 20958 & 20911 & 20911 & \multirow{10}{*}{$50 * 20$} & 129095 & 127348 & 126962 & 128622 & 126719 \\
\hline & 22927 & 22440 & 22591 & 22440 & 22440 & & 122094 & 121208 & 121098 & 122173 & 119600 \\
\hline & 20072 & 19833 & 19968 & 19833 & 19833 & & 121379 & 118051 & 117524 & 118719 & 117241 \\
\hline & 18857 & 18724 & 18769 & 18710 & 18710 & & 124083 & 123061 & 122807 & 123028 & 121319 \\
\hline & 18939 & 18644 & 18749 & 18641 & 18641 & & 122158 & 119920 & 119221 & 121202 & 119099 \\
\hline & 19608 & 19245 & 19245 & 19249 & 19245 & & 124061 & 122369 & 122262 & 123217 & 121343 \\
\hline & 18723 & 18376 & 18377 & 18363 & 18363 & & 126363 & 125609 & 125351 & 125586 & 123807 \\
\hline & 20504 & 20241 & 20377 & 20241 & 20241 & & 126317 & 124543 & 124374 & 125714 & 123054 \\
\hline & 20561 & 20330 & 20330 & 20330 & 20330 & & 125318 & 124059 & 123646 & 124932 & 122765 \\
\hline & 21506 & 21320 & 21323 & 21320 & 21320 & & 127823 & 126582 & 125767 & 126311 & 124970 \\
\hline \multirow{10}{*}{$20 * 20$} & 34119 & 33623 & 33623 & 34975 & 33623 & \multirow{10}{*}{$100 * 5$} & 256789 & 257025 & 257886 & 254762 & 254931 \\
\hline & 31918 & 31604 & 31597 & 32659 & 31587 & & 245609 & 246612 & 246326 & 245315 & 244066 \\
\hline & 34552 & 33920 & 34130 & 34594 & 33920 & & 241013 & 240537 & 241271 & 239777 & 239152 \\
\hline & 32159 & 31698 & 31753 & 32716 & 31661 & & 231365 & 230480 & 230376 & 228872 & 228655 \\
\hline & 34990 & 34593 & 34642 & 35455 & 34557 & & 244016 & 243013 & 243457 & 242245 & 241678 \\
\hline & 32734 & 32637 & 32594 & 33530 & 32564 & & 235793 & 236225 & 236409 & 234082 & 233800 \\
\hline & 33449 & 33038 & 32922 & 33733 & 32922 & & 243741 & 243935 & 243854 & 242122 & 241440 \\
\hline & 32611 & 32444 & 32533 & 33008 & 32412 & & 235171 & 234813 & 234579 & 232755 & 232375 \\
\hline & 34084 & 33625 & 33623 & 34446 & 33600 & & 251291 & 252384 & 253325 & 249959 & 249056 \\
\hline & 32537 & 32317 & 32317 & 33281 & 32262 & & 247491 & 246261 & 246750 & 244275 & 244503 \\
\hline \multirow{10}{*}{$50 * 5$} & 65663 & 65768 & 65546 & 65058 & 64848 & \multirow{10}{*}{$100 * 10$} & 306375 & 305004 & 305376 & 303142 & 301927 \\
\hline & 68664 & 68828 & 68485 & 68298 & 68159 & & 280928 & 279094 & 278921 & 277109 & 277952 \\
\hline & 64378 & 64166 & 64149 & 63577 & 63331 & & 296927 & 297177 & 294239 & 292465 & 291839 \\
\hline & 69795 & 69113 & 69359 & 68571 & 68458 & & 309607 & 306994 & 306739 & 304676 & 304591 \\
\hline & 70841 & 70331 & 70154 & 69698 & 69691 & & 291731 & 290493 & 289676 & 288242 & 288676 \\
\hline & 68084 & 67563 & 67664 & 67138 & 67081 & & 276751 & 276449 & 275932 & 272790 & 273031 \\
\hline & 67186 & 67014 & 66600 & 66338 & 66470 & & 288199 & 286545 & 284846 & 282440 & 282252 \\
\hline & 65582 & 64863 & 65123 & 64638 & 64620 & & 296130 & 297454 & 297400 & 293572 & 293951 \\
\hline & 63968 & 63735 & 63483 & 63227 & 63170 & & 312175 & 309664 & 307043 & 305605 & 305348 \\
\hline & 70273 & 70256 & 69831 & 69195 & 69213 & & 298901 & 296869 & 297182 & 295173 & 295098 \\
\hline
\end{tabular}

suitable for permutation flow shop scheduling by using the LRV rule. This is proposed to convert the continuous encoding in BBO to a discrete job permutation. For initialing the population, The NEH heuristic was combined with the random initialization to initialize the population with certain quality and diversity. In BBO-local search, 
Table 11. Contnd.

\begin{tabular}{cccccc}
\hline \multirow{2}{*}{$\mathbf{n}^{*} \mathbf{m}$} & \multicolumn{5}{c}{ Total flowtime of jobs } \\
\cline { 2 - 6 } & BEST(LR) & M-MMAS & PACO & PSOVNS & HBBO \\
\hline & 383865 & 373756 & 372630 & 374351 & 371828 \\
& 383976 & 383614 & 381124 & 379792 & 377692 \\
$100 * 20$ & 383779 & 380112 & 379135 & 378174 & 376161 \\
& 384854 & 380201 & 380765 & 380899 & 378623 \\
& 383802 & 377268 & 379064 & 376187 & 375685 \\
& 387962 & 381510 & 380464 & 379248 & 378616 \\
& 384839 & 381963 & 382015 & 380912 & 378543 \\
& 397264 & 393617 & 393075 & 392315 & 389896 \\
& 387831 & 385478 & 380359 & 382212 & 380256 \\
& 394861 & 387948 & 388060 & 386013 & 384096 \\
\hline
\end{tabular}

Table 12. Relative performance of five heuristic for mean percent relative increase in total flowtime with respect to the best heuristic solution.

\begin{tabular}{lccccc}
\hline & BES(LR) & M-MAAS & PACO & PSOVNS & HBBO \\
\hline $20 * 5$ & 1.36 & 0.20 & 0.45 & 0.00 & 0.00 \\
$20 * 10$ & 1.43 & 0.05 & 0.32 & 0.002 & 0.00 \\
$20 * 20$ & 1.22 & 0.12 & 0.19 & 2.83 & 0.00 \\
$50 * 5$ & 1.43 & 1.01 & 0.83 & 0.13 & 0.02 \\
$50 * 10$ & 2.42 & 1.43 & 1.17 & 0.19 & 0.09 \\
$50 * 20$ & 2.37 & 1.05 & 0.74 & 1.60 & 0.00 \\
$100 * 5$ & 0.96 & 0.91 & 1.03 & 0.20 & 0.02 \\
$100 * 10$ & 1.54 & 1.13 & 0.85 & 0.08 & 0.06 \\
$100 * 20$ & 2.15 & 0.90 & 0.67 & 0.49 & 0.00 \\
Average & 1.6533 & 0.7556 & 0.6944 & 0.6136 & 0.0211 \\
\hline
\end{tabular}

BBO's migration and mutation can perform a wide global search in the whole solution space. This means that BBO-local search has the ability of obtaining enough subregions over the whole solution space. Then, the fast local search is proposed to enhance the individual of the BBO with a certain probability. Finally, the pairwise based local search is used to enhance the global optimal solution and help the algorithm to escape from local minimum. Experimental results and comparisons show the effectiveness of the proposed HBBO for PFSSP. Moreover, the further work is to study the theoretical aspects as well as the performance of the technique. The other problem is to extend the algorithm to solve other combination problem such as job shop scheduling.

\section{ACKNOWLEDGMENTS}

This research is fully supported by the National Natural Science Foundation of China under Grant Nos.60473042, 60573067,60803102 , and also funded by NSFC Major
Research Program 60496321: Basic Theory and Core Techniques of Non Canonical Knowledge.

\section{REFERENCES}

Bansal SP (1977). Minimizing the sum of completion times of $n$-jobs over M-machines in a flowshop---a branch and bound approach, AllE Trans., 9: 306-311.

Bassem J, Eddaly M (2009). An estimation of distribution algorithm for minimizing the total flowtime in permutation flowshop scheduling problems, Comput. Operat. Res., 36: 2638-2646.

Bhattacharya A, Chattopadhyay P (2010). Biogeography-based optimization for different economic load dispatch problems, IEEE Trans Power Syst., 25: 1064-1077.

Bhattacharya A, Chattopadhyay P (2010). Solving complex economic load dispatch problems using biogeography-based optimization, Expert Syst. Appl., 37: 3605-3615.

Campbell HG, Dudek RA, Smith ML (1970). A heuristic algorithm for the $\mathrm{n}$ job, $\mathrm{m}$ machine sequencing problem, Manage. Sci., 16:630-637.

Cariler J (1978). Ordonnancements a contraintes disjonctives, R.A.I.R.O. Recherche Operationelle/Oper .12: 333-351.

Croce FD, Ghirardi M, Tadei R (2002). An improved branch-and-bound algorithm for the two machine total completion time flow shop problem, Eur. J. Operat. Res., 139: 293-301. 
Croce FD, Narayan V, Tadei R (1996). The two-machine total completion time flow shop problem, Eur. J. Operat. Res., 90 :227-237.

Demirkol E, Mehta S, Uzsoy R (1998), Benchmarks for shop scheduling problems. Eur. J. Operat. Res., 109: 137-141.

Dipak L, Uday K, Chakraborty (2007). An efficient stochastic hybrid heuristic for flowshop scheduling, Eng. Appl. Artif. intel., 20:851-856.

Dong XY, Huang HK (2009), An iterated local search algorithm for the permutation flowshop problem with total flowshop crition, Comput. Operat. Res., 36: 1664-1669.

Framinan JM, Leisten R (2003). An efficient constructive heuristic for flowtime minimization in permutation flow shops, OMEGA. 31: 311317.

Garey MR, Johnson DS (1979). Computers and Intractability: A Guide to the Theory of NP-Completeness, San Francisco, CA: Freeman, pp. $\mathrm{x}+338$. ISBN 0-7167-1045-5.

Gong W, Cai Z, Ling C, Li H (2010). A real-coded biogeography-based optimization with mutation, Appl. Math. Comput., 216: 2749-2758.

Grabowski J, Wodecki M (2004). A very fast tabu search algorithm for the permutation flowshop problem with makespan criterion, Comput. Operat. Res., 31: 1891-1909.

Gupta JND (1972). Heuristic algorithms for multistage flowshop scheduling problem, AlIE Transactions. 4: 11-18.

Ho JC, Chang YL(1991). A new heuristic for the n-job, M-machine flowshop problem, Eur. J. Operat. Res., 52: 194-202.

Ignall E, Schrage L (1965). Application of the branch and bound technique to some flowshop scheduling problem, Operat. Res., 13: 400-412.

Johnson SM (1954). Optimal two-and three-stage production schedules, Naval Res. Logist. Quart., 1: 61-68.

Liu B, Wang L (2007). An Effective PSO-Based Memetic Algorithm for flow shop scheduling, IEEE Transaction Systems, Man, CyberneticsPart B. 37: 18-27.

Liu J, Reeves CR (2001). Constructive and composite heuristic solutions to $P \| \sum C_{i}$ scheduling problem, Eur. J. Operat. Reas., 132: 439-452.

Ma $H$ (2010). An analysis of the equilibrium of migration models for biogeography-based optimization, Inf. Sci., 180: 3444-3464.

Ma H, Simon D (2010). Biogeography-based optimization with blended migration for constrained optimization problems, Gen. Evol. Comput. Conf. Portland, Oregon., 6: 417-418.

Ma H, Simon D (2010). Blended biogeography-based optimization for constrained optimization, Engineering Applications of Artificial Intelligence, 24(3): 517-525.

Murata T, Ishibuchi H, Tanaka H (1996). Genetic algorithms for flowshop scheduling problems, Comput. Operat. Res., 30: 1061-1071.

Nawaz M, Enscore Jr EE, Ham I (1983). A heuristic algorithm for the mmachine, n-job flow shop sequencing problem, OMEGA. 11: 91-95.

Nearchou AC (2004). A novel metaheuristic approach for the flow shop scheduling problem, Eng. Appl. Artif. Intel. 17: 289-300.

Nowicki E, Smutnicki C (1996). A fast tabu search algorithm for the permutation flowshop problem, Eur. J. Operat. Res., 91:160-175.

Ogbu F, Smith D (1990). The application of the simulated annealing algorithm to the solution of the $\mathrm{n} / \mathrm{m} / \mathrm{C}$ max flowshop problem, Comput. Operat. Res., 17: 243-253.

Orhan E, Alper D (2004). A new approach to solve hybrid flow shop scheduling problems by artificial immune system, Future Generat. Comput. Systs., 20: 1083-1095.

Osman I, Potts C (1989). Simulated annealing for permutation flow shop scheduling, OMEGA. 17: 551-557.

Palmer DS (1965). Sequencing jobs through a multistage process in the minimum total time: A quick method of obtaining a near-optimum, Operat. Res. Quart., 16:101-107.
Qian B, wang L (2008).A hybrid differential evolution method for permutation flow-shop scheduling, The International J. Adv. Man. Technol., 38: 757-777.

Rajendran C (1993), Heuristic algorithm for scheduling in a flowshop to minimize total flowtime, Int. J. Prod. Econ., 29: 65-73.

Rajendran C, Ziegler H (2004). Ant-colony algorithms for permutation flowshop scheduling to minimize makespan/total flowtime of jobs, Eur. J. Operat. Res., 155: 426-438.

Reeves C (1995). A genetic algorithm for flowshop sequencing, Comput. Operat. Res., 22: 5-13.

Reeves C, Yamada T (1998). Genetic algorithms, path relinking and the flowshop sequencing problem, Evol. Comput., 6:45-60.

Revees C (1993). Improving the efficiency of tabu search for machine sequencing problem, J. Operat. Res. Soc., 44: 375-382.

Rinnooy KAHG (1976). Machine Scheduling Problems: Classification, Complexity, and Computations, Nijhoff, The Hague, pp.130-194.

Roy P, Ghoshal S, Thakur S (2010). Biogeography-based optimization for economic load dispatch problems, Electric Power Compon. Syst., 38:166-181.

Simon D (2008). Biogeography-Based Optimization. IEEE Trans. Evol. Comput., 12: 702-713.

Stadtler H (2005). Supply chain management and advanced planning basics, overview and challenges, Eur. J. Operat. Res., 163: 575-588.

Stafford EF (1988). On the development of a mixed integer linear programming model for the flowshop sequencing problem, J. Operat. Res. Soc., 39: 1163-1174.

Stützle T (1998). Applying iterated local search to the permutation flowshop problem, Technical Report, AIDA-98-04, Darmstad University of Technology, Computer Science Department, Intellctics Group, Darmstad, Germany.

Stützle T (1998). An ant approach to the flowshop problem, In: Proceedings of the 6th European Congress on Intelligent Techniques and Soft Computing (EUFIT'98), Verlag Mainz, Aachen, Germany, pp. 1560-1564.

Taillard E (1990), Some efficient heuristic methods for the flowshop sequencing problems, Eur. J. Operat. Res., 47:65-74.

Taillard E (1993). Benchmarks for basic scheduling problems. Eur. J. Operat. Res., 64: 278-285.

Tasgetiren MF, Liang YC, Sevkli M, Gencyilmaz G (2007). A particle swarm optimization algorithm for makespan and total flowtime minimization in the permutation flowshop sequencing problem, Eur. J. Operational Res., 177: 1930-1947.

Tasgetiren MF, Liang YC, Sevkli M, Gencyilmaz G (2004). Particle swarm optimization algorithm for makespan and maximum lateness minimization in permutation flowshop sequencing problem. In: Proceedings of the fourth international symposium on intelligent manufacturing systems. Turkey: Sakarya, pp. 431-41.

Tseng LY, Lin YT (2009). A hybrid genetic local search algorithm for the permutation flowshop scheduling problem, Eur. J. operat. Res., 198: 84-92.

Wang L, Wu H, Tang F, Zheng DZ (2005). A hybrid quantum inspired genetic algorithm for flow shop scheduling, Lect Notes Comput. Sci., 3645: 636-644.

Wang L, Zheng DZ (2003). An effective hybrid heuristic for flow shop scheduling, The Int. J. Adv. Man. Technol., 21: 38-44.

Zhang Y, Li XP, Wang Q (2009). Hybrid genetic algorithm for permutation flowshop scheduling problems with total flowtime minimization, Eur. J. Operat. Res., 196: 869-876.

Zheng TM, Yamashiro MS (2010). Solving flow shop scheduling problems by quantum differential evolutionary algorithm, Int J Adv Manuf Technol., DOI 10.1007/s00170-009-2438-4. 